\title{
Medium Effect on the Rotational Barrier of Carbamates and Its Sulfur Congeners
}

\author{
Rodrigo M. Pontes, Ernani A. Basso*, Francisco P. dos Santos.
}

Departamento de Química, Universidade Estadual de Maringá, Av. Colombo, 5790, 87020-900, Maringá-PR, Brazil.

* eabasso@uem.br 


\section{Table of Contents}

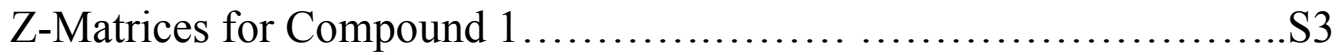

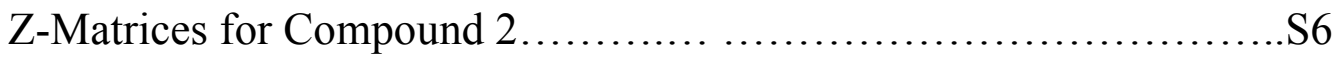

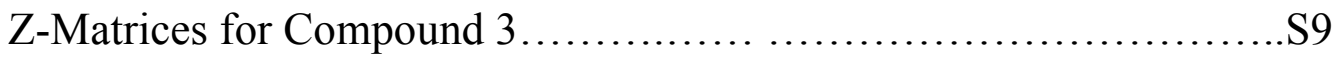

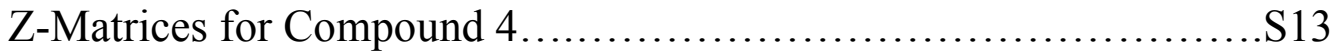

NMR Data on Compound 1.......................................S17

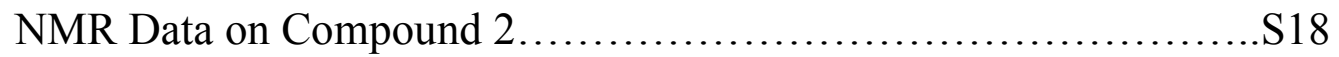

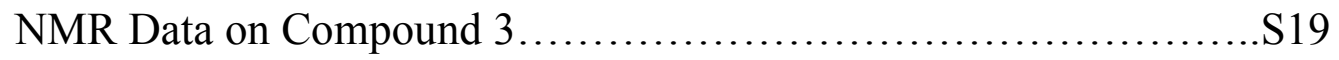

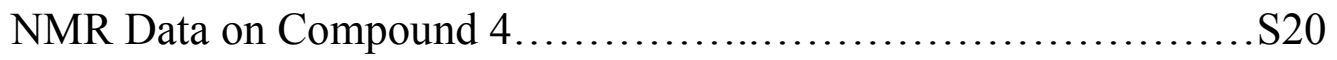

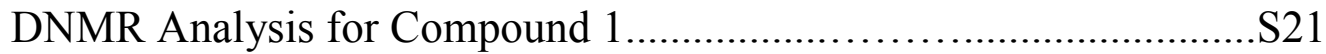

Force Field Parameters for MD Simulations.......................S22 


\section{Methyl N,N-Dimethylcarbamate (1) GS 6-311+G(2d,p)}

C

$0,1, r 2$

$\mathrm{C}, 2, \mathrm{r3}, 1, \mathrm{a} 3$

$\mathrm{N}, 3, \mathrm{r} 4,2, \mathrm{a} 4,1, \mathrm{~d} 4,0$

$C, 4, r 5,3, a 5,2, d 5,0$

$0,3, r 6,2, a 6,1, d 6,0$

$\mathrm{C}, 4, \mathrm{r} 7,3, \mathrm{a} 7,2, \mathrm{~d} 7,0$

$\mathrm{H}, 1, \mathrm{r} 8,2, \mathrm{a} 8,3, \mathrm{~d} 8,0$

$\mathrm{H}, 1, \mathrm{r9}, 2, \mathrm{a} 9,3, \mathrm{~d} 9,0$

$\mathrm{H}, 1, \mathrm{r10}, 2, \mathrm{a} 10,3, \mathrm{~d} 10,0$

$\mathrm{H}, 7, \mathrm{r} 11,4, \mathrm{a} 11,3, \mathrm{~d} 11,0$

$\mathrm{H}, 7, \mathrm{r} 12,4, \mathrm{a} 12,3, \mathrm{~d} 12, \mathrm{O}$

$\mathrm{H}, 7, \mathrm{r} 13,4, \mathrm{a} 13,3, \mathrm{~d} 13, \mathrm{O}$

$\mathrm{H}, 5, \mathrm{r} 14,4, \mathrm{a} 14,3, \mathrm{~d} 14,0$

$\mathrm{H}, 5, \mathrm{r} 15,4, \mathrm{a} 15,3, \mathrm{~d} 15,0$

$\mathrm{H}, 5, \mathrm{r} 16,4, \mathrm{a} 16,3, \mathrm{~d} 16,0$

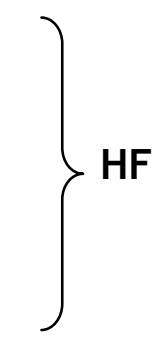

$r 2=1.41398667$

$r 3=1.32566882$

a $3=116.37200204$

$r 4=1.3507289$

$\mathrm{a}_{4}=112.61288012$

$d 4=-177.78685488$

$r 5=1.4476427$

a $5=122.71188196$

$d 5=-10.96772162$

$r 6=1.19372954$

$a 6=122.66210236$

$d 6=1.32145108$

$r 7=1.44704498$

a $7=118.2953979$

$d 7=-171.78332434$

$r 8=1.07995929$

a $8=110.62721011$

$d 8=60.87372287$

$r 9=1.07977533$

a $9=110.64791757$

$\mathrm{d} 9=-60.38731682$

r $10=1.07905628$

a1 $0=105.62810119$

$\mathrm{d} 10=-179.78175674$

$r 11=1.08770894$

$\mathrm{a} 11=111.32508573$

$\mathrm{d} 11=96.94823916$

$r 12=1.07616445$

a12 $=109.73103611$

$\mathrm{d} 12=-23.60122238$

$r 13=1.08348594$

a $13=109.2726163$

d13 $=-143.32451833$

$r 14=1.07613518$

a14 $=110.84890705$

d1 $4=33.9370355$

$r 15=1.08773718$

a1 $5=111.71828113$

d1 $5=-87.64628481$

$r 16=1.08286719$

a $16=108.56986166$

d1 $6=153.07801765$

$E=-361.011323$

$\# i=0$
C

$0,1, r 2$

$\mathrm{C}, 2, \mathrm{r3}, 1, \mathrm{a} 3$

$\mathrm{N}, 3, \mathrm{r} 4,2, \mathrm{a} 4,1, \mathrm{~d} 4,0$

$C, 4, r 5,3, a 5,2, d 5,0$

$0,3, r 6,2, a 6,1, d 6,0$

C, $4, r 7,3, a 7,2, d 7,0$

$\mathrm{H}, 1, \mathrm{r} 8,2, \mathrm{a} 8,3, \mathrm{~d} 8,0$

$\mathrm{H}, 1, \mathrm{r} 9,2, \mathrm{a} 9,3, \mathrm{~d} 9,0$

$\mathrm{H}, 1, \mathrm{r} 10,2, \mathrm{a} 10,3, \mathrm{~d} 10,0$

$\mathrm{H}, 7, \mathrm{r} 11,4, \mathrm{a} 11,3, \mathrm{~d} 11,0$

$\mathrm{H}, 7, \mathrm{r12}, 4, \mathrm{a} 12,3, \mathrm{~d} 12, \mathrm{O}$

$\mathrm{H}, 7, \mathrm{r13}, 4, \mathrm{a} 13,3, \mathrm{~d} 13, \mathrm{O}$

$\mathrm{H}, 5, \mathrm{r14}, 4, \mathrm{a} 14,3, \mathrm{~d} 14,0$

$\mathrm{H}, 5, \mathrm{r} 15,4, \mathrm{a} 15,3, \mathrm{~d} 15,0$

$\mathrm{H}, 5, \mathrm{r} 16,4, \mathrm{a} 16,3, \mathrm{~d} 16,0$

$r 2=1.43461022$

$r 3=1.36118763$

a $3=114.66597218$

$r 4=1.36215754$

a $4=112.06684473$

$\mathrm{d} 4=-178.79391$

$r 5=1.45354115$

a $5=124.30973892$

$\mathrm{d} 5=-3.2938553$

$r 6=1.2160609$

a $6=122.88023959$

$\mathrm{d} 6=0.83780338$

$r 7=1.45258372$

a $7=118.89612304$

$d 7=-176.85152912$

$r 8=1.08970153$

a $8=110.6782902$

$d 8=61.05324943$

$r 9=1.08951789$

a $9=110.67234132$

d $9=-60.01823254$

r $10=1.08773228$

a $10=105.31005819$

d1 $0=-179.50515335$

$r 11=1.09554437$

$\mathrm{a} 11=110.7173896$

$\mathrm{d} 11=113.26135478$

$r 12=1.08587491$

a $12=109.12493947$

d12 $=-6.90840866$

$r 13=1.0940558$

a $13=110.11977964$

d13 $=-126.98690395$

$r 14=1.08435191$

a $14=110.4563299$

d14 $=12.32697354$

$r 15=1.09604042$

a $15=110.88596166$

d15 $=-108.46664482$

$r 16=1.09397323$

a1 $6=109.59403364$

d1 $6=132.2776719$
B3LYP

\author{
$E=-363.175947$ \\ $\# i=0$
}




\section{Methyl N,N-Dimethylcarbamate (1) TS1 6-311+G(2d,p)}

C

$0,1,22$

C, $2, r 3,1$, a 3

$\mathrm{N}, 3, \mathrm{r} 4,2, \mathrm{a} 4,1, \mathrm{~d} 4,0$

$\mathrm{C}, 4, \mathrm{r} 5,3, \mathrm{a} 5,2, \mathrm{~d} 5,0$

$0,3, r 6,2, a 6,1, d 6,0$

$\mathrm{C}, 4, \mathrm{r} 7,3, \mathrm{a} 7,2, \mathrm{~d} 7,0$

$\mathrm{H}, 1, \mathrm{r} 8,2, \mathrm{a} 8,3, \mathrm{~d} 8,0$

$\mathrm{H}, 1, \mathrm{r9}, 2, \mathrm{a} 9,3, \mathrm{~d} 9,0$

$\mathrm{H}, 1, \mathrm{r} 10,2, \mathrm{a} 10,3, \mathrm{~d} 10,0$

$\mathrm{H}, 7, \mathrm{r} 11,4, \mathrm{a} 11,3, \mathrm{~d} 11,0$

$\mathrm{H}, 7, \mathrm{r12}, 4, \mathrm{a} 12,3, \mathrm{~d} 12,0$

$\mathrm{H}, 7, \mathrm{r13}, 4, \mathrm{a} 13,3, \mathrm{~d} 13,0$

$\mathrm{H}, 5, \mathrm{r} 14,4, \mathrm{a} 14,3, \mathrm{~d} 14,0$

$\mathrm{H}, 5, \mathrm{r} 15,4, \mathrm{a} 15,3, \mathrm{~d} 15, \mathrm{O}$

$\mathrm{H}, 5, \mathrm{r} 16,4, \mathrm{a} 16,3, \mathrm{~d} 16,0$

$r 2=1.41398667$

$r 3=1.32566882$

a $3=116.37200204$

$r 4=1.3507289$

$a_{4}=112.61288012$

$\mathrm{d} 4=-177.78685488$

$r 5=1.4476427$

a $5=122.71188196$

$\mathrm{d} 5=-10.96772162$

$r 6=1.19372954$

a $6=122.66210236$

$d 6=1.32145108$

$r 7=1.44704498$

a $7=118.2953979$

$\mathrm{d} 7=-171.78332434$

$r 8=1.07995929$

$a 8=110.62721011$

$\mathrm{d} 8=60.87372287$

$r 9=1.07977533$

a $9=110.64791757$

$d 9=-60.38731682$

$r 10=1.07905628$

a1 $0=105.62810119$

d1 $0=-179.78175674$

$r 11=1.08770894$

$\mathrm{a} 11=111.32508573$

$\mathrm{d} 11=96.94823916$

$r 12=1.07616445$

a12 $=109.73103611$

$\mathrm{d} 12=-23.60122238$

$r 13=1.08348594$

a $13=109.2726163$

d13 $=-143.32451833$

$r 14=1.07613518$

a14 $=110.84890705$

d14 $=33.9370355$

$r 15=1.08773718$

a $15=111.71828113$

$\mathrm{d} 15=-87.64628481$

$r 16=1.08286719$

a $16=108.56986166$

d1 $6=153.07801765$

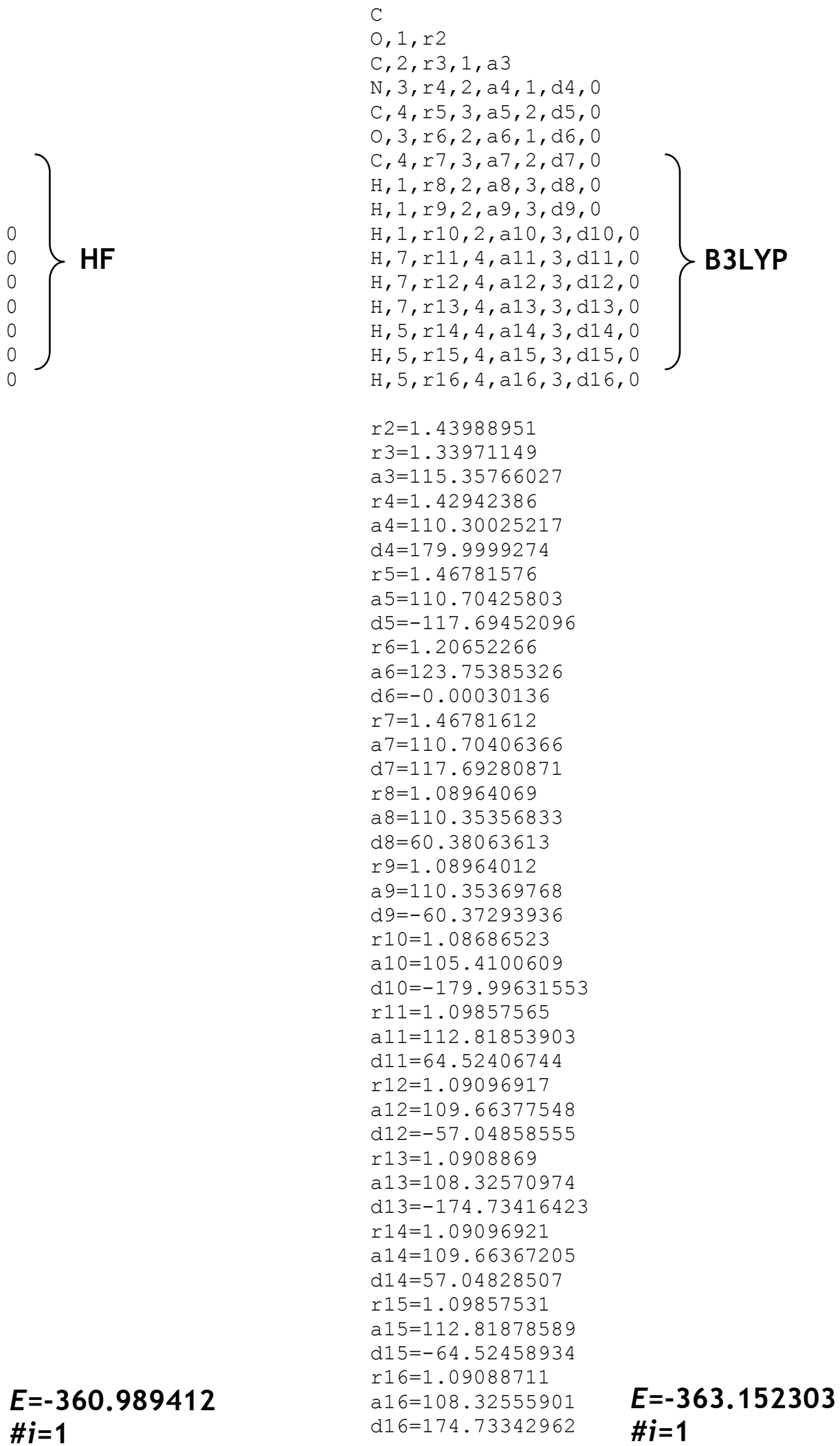

C

C, $2, r 3,1$, a 3

$\mathrm{N}, 3, \mathrm{r} 4,2, \mathrm{a} 4,1, \mathrm{~d} 4,0$

$\mathrm{C}, 4, \mathrm{r} 5,3, \mathrm{a} 5,2, \mathrm{~d} 5,0$

$\mathrm{H}, 1, \mathrm{r10}, 2$, a10, 3, d10,

$\mathrm{H}, 7, \mathrm{r} 11,4, \mathrm{a} 11,3, \mathrm{~d} 11,0$

$\mathrm{H}, 7, \mathrm{r} 12,4, \mathrm{a} 12,3, \mathrm{~d} 12,0$

$\mathrm{H}, 7, \mathrm{r13}, 4, \mathrm{a} 13,3, \mathrm{~d} 13, \mathrm{O}$

$\mathrm{H}, 5, \mathrm{r15}, 4, \mathrm{a} 15,3, \mathrm{~d} 15,0$

$r 2=1.43988951$

$r 3=1.33971149$

a $3=115.35766027$

$r 4=1.42942386$

$4=110 \cdot 3002521$

a $5=110.70425803$

$\mathrm{d} 5=-117.69452096$

$6=1.20652266$

$r 7=1.46781612$

a $7=110.70406366$

$\mathrm{d} 7=117.69280871$

$r 8=1.08964069$

a $9=110.35369768$

d $9=-60.37293936$

a $11=112.81853903$

$\mathrm{d} 11=64.52406744$

$r 12=1.09096917$

$12=109.66377548$

$r 13=1.0908869$

a $13=108.32570974$

$r 14=1.09096921$

a $14=109.66367205$

d14 $=57.04828507$

$=1.09857531$

r16=1.09088711

d1 $6=174.73342962$
$\# i=1$ 


\section{Methyl N,N-Dimethylcarbamate (1) TS2 6-311+G(2d,p)}

C

$0,1, r 2$

$\mathrm{C}, 2, \mathrm{r3}, 1, \mathrm{a} 3$

$\mathrm{N}, 3, \mathrm{r} 4,2, \mathrm{a} 4,1, \mathrm{~d} 4,0$

$\mathrm{C}, 4, \mathrm{r} 5,3, \mathrm{a} 5,2, \mathrm{~d} 5,0$

$0,3, r 6,2, a 6,1, d 6,0$

$\mathrm{C}, 4, \mathrm{r} 7,3, \mathrm{a} 7,2, \mathrm{~d} 7,0$

$\mathrm{H}, 1, \mathrm{r} 8,2, \mathrm{a} 8,3, \mathrm{~d} 8,0$

$\mathrm{H}, 1, \mathrm{r} 9,2, \mathrm{a} 9,3, \mathrm{~d} 9, \mathrm{O}$

$\mathrm{H}, 1, \mathrm{r} 10,2, \mathrm{a} 10,3, \mathrm{~d} 10,0$

$\mathrm{H}, 7, \mathrm{r} 11,4, \mathrm{a} 11,3, \mathrm{~d} 11,0$

$\mathrm{H}, 7, \mathrm{r} 12,4, \mathrm{a} 12,3, \mathrm{~d} 12, \mathrm{O}$

$\mathrm{H}, 7, \mathrm{r13}, 4, \mathrm{a} 13,3, \mathrm{~d} 13, \mathrm{O}$

$\mathrm{H}, 5, \mathrm{r} 14,4, \mathrm{a} 14,3, \mathrm{~d} 14, \mathrm{O}$

$\mathrm{H}, 5, \mathrm{r} 15,4, \mathrm{a} 15,3, \mathrm{~d} 15,0$

$\mathrm{H}, 5, \mathrm{r16}, 4, \mathrm{a} 16,3, \mathrm{~d} 16,0$

$r 2=1.41487755$

$r 3=1.32358989$

a $3=117.02902384$

$r 4=1.4107451$

$a_{4}=112.72702033$

$d 4=179.99883032$

$r 5=1.45460261$

a $5=112.58966954$

$d 5=64.50297568$

$r 6=1.17848914$

$a 6=123.10895027$

$d 6=0.0021583$

$r 7=1.45460341$

a $7=112.58797526$

$d 7=-64.53977327$

$r 8=1.08034304$

$a 8=110.4819771$

$d 8=60.51282428$

$r 9=1.08034226$

a $9=110.48246092$

$\mathrm{d} 9=-60.50978724$

r $10=1.07875526$

a $10=105.88912862$

d1 $0=-179.99872211$

$r 11=1.08858219$

$\mathrm{a} 11=113.03497415$

$\mathrm{d} 11=70.32217219$

$r 12=1.08286807$

a12 $=109.68401273$

$\mathrm{d} 12=-51.41402955$

$r 13=1.08238375$

a13 $=108.40695942$

d13 $=-168.97029726$

$r 14=1.08286842$

a1 $4=109.68440379$

d1 $4=51.3882378$

$r 15=1.08857994$

a $15=113.03589443$

$\mathrm{d} 15=-70.34771465$

$r 16=1.08238421$

a1 $6=108.40609824$

d1 $6=168.94395882$

\author{
C
}

$0,1, r 2$

C, $2, r 3,1$, a 3

$\mathrm{N}, 3, \mathrm{r} 4,2, \mathrm{a} 4,1, \mathrm{~d} 4,0$

C, 4, r5 $, 3, a 5,2, d 5,0$

$0,3, r 6,2, a 6,1, d 6,0$

$\mathrm{C}, 4, \mathrm{r} 7,3, \mathrm{a} 7,2, \mathrm{~d} 7,0$

$\mathrm{H}, 1, \mathrm{r} 8,2, \mathrm{a} 8,3, \mathrm{~d} 8,0$

$\mathrm{H}, 1, \mathrm{rg}, 2, \mathrm{a} 9,3, \mathrm{~d} 9, \mathrm{O}$

$\mathrm{H}, 1, \mathrm{r} 10,2, \mathrm{a} 10,3, \mathrm{~d} 10,0$

$\mathrm{H}, 7, \mathrm{r} 11,4, \mathrm{a} 11,3, \mathrm{~d} 11,0$

$\mathrm{H}, 7, \mathrm{r} 12,4, \mathrm{a} 12,3, \mathrm{~d} 12, \mathrm{O}$

$\mathrm{H}, 7, \mathrm{r13}, 4, \mathrm{a} 13,3, \mathrm{~d} 13,0$

$\mathrm{H}, 5, \mathrm{r} 14,4, \mathrm{a} 14,3, \mathrm{~d} 14,0$

$\mathrm{H}, 5, \mathrm{r} 15,4, \mathrm{a} 15,3, \mathrm{~d} 15,0$

$\mathrm{H}, 5, \mathrm{r} 16,4, \mathrm{a} 16,3, \mathrm{~d} 16,0$

$r 2=1.43879174$

$r 3=1.35274366$

a $3=115.79088986$

$r 4=1.42489946$

a $4=112.12881643$

d $4=179.99942753$

$r 5=1.46523147$

a $5=112.76979047$

$\mathrm{d} 5=64.53489911$

$r 6=1.20091428$

a $6=123.2462744$

$\mathrm{d} 6=0$.

$r 7=1.4652304$

a $7=112.76944025$

$\mathrm{d} 7=-64.53820809$

$r 8=1.08965446$

$a 8=110.33154344$

$\mathrm{d} 8=60.34553164$

$r 9=1.08965473$

a $9=110.33129554$

$d 9=-60.34907153$

$r 10=1.08729442$

a1 $0=105.59732292$

d1 $0=179.99836389$

$r 11=1.09899164$

a $11=113.22859845$

d11 $=70.42135417$

$r 12=1.09086551$

a12=109.59684637

d12 $=-51.44190387$

$r 13=1.09139058$

a13 $=108.31243015$

d13 $=-168.91505772$

$r 14=1.09086499$

a14=109.59684448

d1 $4=51.43639705$

$r 15=1.09899204$

a1 $5=113.22880547$

d15 $=-70.4270311$

$r 16=1.09139112$

a1 $6=108.31238201$

d1 $6=168.90957892$

$E=-363.150689$

$\# i=1$
B3LYP
$\# i=1$ 


\section{S-Methyl N,N-Dimethylthiocarbamate (2) GS $6-311+G(2 d, p)$}

C

$\mathrm{S}, 1, \mathrm{r} 2$

C, $2, r 3,1$, a 3

$\mathrm{N}, 3, \mathrm{r} 4,2, \mathrm{a} 4,1, \mathrm{~d} 4,0$

$\mathrm{C}, 4, \mathrm{r} 5,3, \mathrm{a} 5,2, \mathrm{~d} 5,0$

$0,3, r 6,2, a 6,1, d 6,0$

$\mathrm{C}, 4, \mathrm{r} 7,3, \mathrm{a} 7,2, \mathrm{~d} 7,0$

$\mathrm{H}, 1, \mathrm{r} 8,2, \mathrm{a} 8,3, \mathrm{~d} 8,0$

$\mathrm{H}, 1, \mathrm{r} 9,2, \mathrm{a} 9,3, \mathrm{~d} 9, \mathrm{O}$

$\mathrm{H}, 1, \mathrm{r} 10,2, \mathrm{a} 10,3, \mathrm{~d} 10,0$

$\mathrm{H}, 7, \mathrm{r} 11,4, \mathrm{a} 11,3, \mathrm{~d} 11, \mathrm{O}$

$\mathrm{H}, 7, \mathrm{r12}, 4, \mathrm{a} 12,3, \mathrm{~d} 12, \mathrm{O}$

$\mathrm{H}, 7, \mathrm{r13}, 4, \mathrm{a} 13,3, \mathrm{~d} 13, \mathrm{O}$

$\mathrm{H}, 5, \mathrm{r} 14,4, \mathrm{a} 14,3, \mathrm{~d} 14, \mathrm{O}$

$\mathrm{H}, 5, \mathrm{r} 15,4, \mathrm{a} 15,3, \mathrm{~d} 15,0$

$\mathrm{H}, 5, \mathrm{r} 16,4, \mathrm{a} 16,3, \mathrm{~d} 16,0$

Variables:

$r 2=1.80858155$

$r 3=1.79512795$

a $3=99.26221766$

$r 4=1.34965289$

a $4=115.92943814$

$\mathrm{d} 4=-178.04935735$

$r 5=1.44705957$

a $5=123.62197255$

$d 5=-9.68416134$

$r 6=1.19253876$

a $6=120.07337439$

$d 6=1.39488593$

$r 7=1.4509602$

a $7=118.21989386$

$d 7=-172.35869182$

$r 8=1.07777981$

$a 8=110.43923796$

$d 8=61.4272504$

$r 9=1.07767304$

a $9=110.40109744$

$\mathrm{d} 9=-60.57538636$

$r 10=1.08185545$

a1 $0=105.61584744$

d1 $0=-179.58791669$

$r 11=1.08712129$

a11 $=111.06340498$

$\mathrm{d} 11=98.76729671$

$r 12=1.07578683$

a12 $=109.68414763$

$\mathrm{d} 12=-21.68821689$

$r 13=1.08321772$

a13 $=109.22707512$

d13 $=-141.48012349$

$r 14=1.07877645$

a14 $=111.37952272$

$\mathrm{d} 14=38.45291631$

$r 15=1.08663235$

a $15=112.00691504$

$\mathrm{d} 15=-83.81149951$

$r 16=1.0818768$

a1 $6=108.43848081$

d16=156.54164141

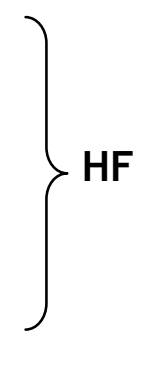

C

$\mathrm{S}, 1, \mathrm{r} 2$

$\mathrm{C}, 2, \mathrm{r} 3,1, \mathrm{a} 3$

$\mathrm{N}, 3, \mathrm{r} 4,2, \mathrm{a} 4,1, \mathrm{~d} 4,0$

$\mathrm{C}, 4, \mathrm{r} 5,3, \mathrm{a} 5,2, \mathrm{~d} 5,0$

$0,3, r 6,2, a 6,1, d 6,0$

$\mathrm{C}, 4, \mathrm{r} 7,3, \mathrm{a} 7,2, \mathrm{~d} 7,0$

$\mathrm{H}, 1, \mathrm{r} 8,2, \mathrm{a} 8,3, \mathrm{~d} 8,0$

$\mathrm{H}, 1, \mathrm{r9}, 2, \mathrm{a} 9,3, \mathrm{~d} 9, \mathrm{O}$

$\mathrm{H}, 1, \mathrm{r} 10,2, \mathrm{a} 10,3, \mathrm{~d} 10,0$

$\mathrm{H}, 7, \mathrm{r} 11,4, \mathrm{a} 11,3, \mathrm{~d} 11,0$

$\mathrm{H}, 7, \mathrm{r12}, 4, \mathrm{a} 12,3, \mathrm{~d} 12, \mathrm{O}$

$\mathrm{H}, 7, \mathrm{r13}, 4, \mathrm{a} 13,3, \mathrm{~d} 13,0$

$\mathrm{H}, 5, \mathrm{r} 14,4, \mathrm{a} 14,3, \mathrm{~d} 14,0$

$\mathrm{H}, 5, \mathrm{r15}, 4, \mathrm{a} 15,3, \mathrm{~d} 15,0$

$\mathrm{H}, 5, \mathrm{r} 16,4, \mathrm{a} 16,3, \mathrm{~d} 16,0$ J

Variables:

$r 2=1.82019156$

$r 3=1.82135846$

a $3=98.05207001$

$r 4=1.36287262$

a $4=115.06575397$

$\mathrm{d} 4=-177.13888427$

$r 5=1.45253087$

a $5=124.11610651$

$\mathrm{d} 5=-3.98829002$

$r 6=1.21545047$

a $6=120.74774894$

$d 6=2.81197372$

$r 7=1.45717732$

a $7=118.74978512$

$d 7=-173.76517717$

$r 8=1.08698903$

$\mathrm{a} 8=110.50059764$

$\mathrm{d} 8=63.34621709$

$r 9=1.08668581$

a $9=110.19637963$

$d 9=-58.40312614$

$r 10=1.0896867$

a $10=105.62672712$

d1 $0=-177.52822006$

$r 11=1.09532372$

$\mathrm{a} 11=110.72628408$

$\mathrm{d} 11=109.68339067$

$r 12=1.08584069$

a $12=109.0090486$

$\mathrm{d} 12=-10.41529239$

$r 13=1.09307435$

a13 $=109.87057439$

$\mathrm{d} 13=-130.42600542$

$r 14=1.08810874$

a $14=110.92371034$

d1 $4=28.64844375$

$r 15=1.09564896$

a $15=111.87699725$

d15 $=-93.10276707$

$r 16=1.09192492$

a $16=108.89049789$

d1 $6=147.02423655$

B3LYP

$E=-683.676750$

$\# \mathbf{i}=0$
$E=-686.146239$

$\# i=0$ 


\section{S-Methyl N,N-Dimethylthiocarbamate (2) TS1 6-311+G(2d,p)}

C

$\mathrm{S}, 1, \mathrm{r} 2$

$\mathrm{C}, 2, \mathrm{r3}, 1, \mathrm{a} 3$

$0,3, \mathrm{r} 4,2, \mathrm{a} 4,1, \mathrm{~d} 4,0$

$\mathrm{N}, 3, \mathrm{r} 5,2, \mathrm{a} 5,1, \mathrm{~d} 5,0$

$C, 5, r 6,3, a 6,2, d 6,0$

$\mathrm{C}, 5, \mathrm{r} 7,3, \mathrm{a} 7,2, \mathrm{~d} 7,0$

$\mathrm{H}, 1, \mathrm{r} 8,2, \mathrm{a} 8,3, \mathrm{~d} 8,0$

$\mathrm{H}, 1, \mathrm{rg}, 2, \mathrm{a} 9,3, \mathrm{~d} 9, \mathrm{O}$

$\mathrm{H}, 1, \mathrm{r} 10,2, \mathrm{a} 10,3, \mathrm{~d} 10,0$

$\mathrm{H}, 6, \mathrm{r} 11,5, \mathrm{a} 11,3, \mathrm{~d} 11,0$

$\mathrm{H}, 6, \mathrm{r} 12,5, \mathrm{a} 12,3, \mathrm{~d} 12, \mathrm{O}$

$\mathrm{H}, 6, \mathrm{r13}, 5, \mathrm{a} 13,3, \mathrm{~d} 13, \mathrm{O}$

$\mathrm{H}, 7, \mathrm{r} 14,5, \mathrm{a} 14,3, \mathrm{~d} 14,0$

$\mathrm{H}, 7, \mathrm{r} 15,5, \mathrm{a} 15,3, \mathrm{~d} 15,0$

$\mathrm{H}, 7, \mathrm{r16}, 5, \mathrm{a} 16,3, \mathrm{~d} 16,0)$ Variables:

$r 2=1.80986985$

$r 3=1.76130094$

a $3=100.93279907$

$r 4=1.17961984$

a $4=123.99154304$

$d 4=0.00351015$

$r 5=1.41959128$

a $5=110.78831674$

$\mathrm{d} 5=-179.99732911$

$r 6=1.45787893$

a $6=111.12499976$

$d 6=116.93125261$

$r 7=1.45788033$

a $7=111.1253157$

$d 7=-116.94883069$

$r 8=1.07887148$

$a 8=110.38030176$

$d 8=60.89827541$

$r 9=1.07887365$

a $9=110.3799796$

$\mathrm{d} 9=-60.88705016$

$r 10=1.0815625$

a $10=106.38044786$

d1 $0=-179.99444371$

$r 11=1.08758639$

$\mathrm{a} 11=112.56461669$

$\mathrm{d} 11=66.20787177$

$r 12=1.08275892$

a12 $=109.66269815$

$\mathrm{d} 12=-55.09817723$

$r 13=1.08217509$

a $13=108.45541743$

d13 $=-172.9493931$

$r 14=1.08275821$

a1 $4=109.66243469$

d1 $4=55.0976558$

$r 15=1.08758573$

a $15=112.56545439$

$\mathrm{d} 15=-66.20836599$

$r 16=1.08217522$

a1 $6=108.45511493$

d1 $6=172.94846858$
C

$\mathrm{S}, 1, \mathrm{r} 2$

$\mathrm{C}, 2, \mathrm{r} 3,1, \mathrm{a} 3$

$0,3, r 4,2, a 4,1, d 4,0$

$\mathrm{N}, 3, \mathrm{r} 5,2, \mathrm{a} 5,1, \mathrm{~d} 5,0$

$c, 5, r 6,3, a 6,2, d 6,0$

C, 5, r7 $, 3, a 7,2, d 7,0$

$\mathrm{H}, 1, \mathrm{r} 8,2, \mathrm{a} 8,3, \mathrm{~d} 8,0$

$\mathrm{H}, 1, \mathrm{rg}, 2, \mathrm{a} 9,3, \mathrm{~d} 9,0$

$\mathrm{H}, 1, \mathrm{r} 10,2, \mathrm{a} 10,3, \mathrm{~d} 10,0$

$\mathrm{H}, 6, \mathrm{r} 11,5, \mathrm{a} 11,3, \mathrm{~d} 11,0$

$\mathrm{H}, 6, \mathrm{r} 12,5, \mathrm{a} 12,3, \mathrm{~d} 12, \mathrm{O}$

$\mathrm{H}, 6, \mathrm{r13}, 5, \mathrm{a} 13,3, \mathrm{~d} 13,0$

$\mathrm{H}, 7, \mathrm{r14}, 5, \mathrm{a} 14,3, \mathrm{~d} 14, \mathrm{O}$

$\mathrm{H}, 7, \mathrm{r} 15,5, \mathrm{a} 15,3, \mathrm{~d} 15, \mathrm{O}$

$\mathrm{H}, 7, \mathrm{r} 16,5, \mathrm{a} 16,3, \mathrm{~d} 16,0$

$r 2=1.82401$

$r 3=1.76906$

$r 4=1.20434$

a $4=124.83944$

$\mathrm{d} 4=0.06837$

$r 5=1.43764$

a $5=109.34529$

$\mathrm{d} 5=-180.01268$

$r 6=1.46881$

a $6=111.09605$

$d 6=117.13607$

$r 7=1.46889$

a $7=111.10694$

$\mathrm{d} 7=-117.20927$

$r 8=1.08776$

$\mathrm{a} 8=110.12302$

$\mathrm{d} 8=60.81738$

$r 9=1.08775$

a $9=110.11377$

d $9=-60.47187$

$r 10=1.0893$

a $10=106.58081$

d10 $=-179.82627$

$r 11=1.09795$

$\mathrm{a} 11=112.75023$

$\mathrm{d} 11=66.03797$

$r 12=1.0908$

a $12=109.53397$

$\mathrm{d} 12=-55.36044$

$r 13=1.09101$

a $13=108.38925$

$\mathrm{d} 13=-173.20153$

$r 14=1.0908$

a $14=109.53164$

$\mathrm{d} 14=55.38546$

$r 15=1.09796$

a $15=112.74928$

$\mathrm{d} 15=-66.00829$

$r 16=1.09102$

a $16=108.40515$

d16 $=173.22655$ a $3=100.40762$

B3LYP

$E=-683.636562$

$\# i=1$
$E=-686.125924$

$\# i=1$ 


\section{S-Methyl N,N-Dimethylthiocarbamate (2) TS2 6-311+G(2d,p)}

C

$\mathrm{S}, 1, \mathrm{r} 2$

$\mathrm{C}, 2, \mathrm{r3}, 1, \mathrm{a} 3$

$\mathrm{N}, 3, \mathrm{r} 4,2, \mathrm{a} 4,1, \mathrm{~d} 4, \mathrm{O}$

$\mathrm{C}, 4, \mathrm{r} 5,3, \mathrm{a} 5,2, \mathrm{~d} 5,0$

$0,3, r 6,2, a 6,1, d 6,0$

$\mathrm{C}, 4, \mathrm{r} 7,3, \mathrm{a} 7,2, \mathrm{~d} 7,0$

$\mathrm{H}, 1, \mathrm{r} 8,2, \mathrm{a} 8,3, \mathrm{~d} 8, \mathrm{O}$

$\mathrm{H}, 1, \mathrm{r} 9,2, \mathrm{a} 9,3, \mathrm{~d} 9, \mathrm{O}$

$\mathrm{H}, 1, \mathrm{r} 10,2, \mathrm{a} 10,3, \mathrm{~d} 10,0$

$\mathrm{H}, 7, \mathrm{r} 11,4, \mathrm{a} 11,3, \mathrm{~d} 11, \mathrm{O}$

$\mathrm{H}, 7, \mathrm{r} 12,4, \mathrm{a} 12,3, \mathrm{~d} 12,0$

$\mathrm{H}, 7, \mathrm{r13}, 4, \mathrm{a} 13,3, \mathrm{~d} 13, \mathrm{O}$

$\mathrm{H}, 5, \mathrm{r} 14,4, \mathrm{a} 14,3, \mathrm{~d} 14, \mathrm{O}$

$\mathrm{H}, 5, \mathrm{r} 15,4, \mathrm{a} 15,3, \mathrm{~d} 15,0$

$\mathrm{H}, 5, \mathrm{r} 16,4, \mathrm{a} 16,3, \mathrm{~d} 16,0$

Variables:

$r 2=1.80995024$

$r 3=1.78824471$

a $3=100.61502066$

$r 4=1.41512083$

a $4=115.44064502$

$d 4=179.97086166$

$r 5=1.45374286$

a $5=114.21205663$

$\mathrm{d} 5=66.66967681$

$r 6=1.17540228$

a $6=121.43460763$

$d 6=-0.02691269$

$r 7=1.45373881$

a $7=114.21066888$

$r 8=1.07836001$

a $8=110.2624685$

$\mathrm{d} 8=60.93586616$

$r 9=1.07834608$

a $9=110.25728845$

$\mathrm{d} 9=-60.79055296$

$r 10=1.08139855$

a $10=106.2146199$

d1 $0=-179.92624919$

$r 11=1.0890602$

$\mathrm{a} 11=113.39871134$

$\mathrm{d} 11=74.95585904$

$r 12=1.08201581$

a12 $=109.75955128$

$\mathrm{d} 12=-46.85130158$

$r 13=1.08258584$

a $13=108.29063865$

d13 $=-164.53643532$

$r 14=1.08201608$

a1 $4=109.76008782$

d1 $4=46.8624081$

$r 15=1.08906212$

a $15=113.40012563$

$\mathrm{d} 15=-74.94524823$

$r 16=1.0825855$

a $16=108.2907891$

d1 $6=164.54730514$

$d 7=-66.68462903$
(2)

$\mathrm{S}, 1, \mathrm{r} 2$

$\mathrm{C}, 2, \mathrm{r} 3,1, \mathrm{a} 3$

$\mathrm{N}, 3, \mathrm{r} 4,2, \mathrm{a} 4,1, \mathrm{~d} 4,0$

$\mathrm{C}, 4, \mathrm{r} 5,3, \mathrm{a} 5,2, \mathrm{~d} 5,0$

$0,3, r 6,2, a 6,1, d 6,0$

$\mathrm{C}, 4, \mathrm{r} 7,3, \mathrm{a} 7,2, \mathrm{~d} 7,0$

$\mathrm{H}, 1, \mathrm{r} 8,2, \mathrm{a} 8,3, \mathrm{~d} 8,0$

$\mathrm{H}, 1, \mathrm{rg}, 2$, a 9, 3, d9, 0

$\mathrm{H}, 1, \mathrm{r} 10,2, \mathrm{a} 10,3, \mathrm{~d} 10,0$

$\mathrm{H}, 7, \mathrm{r} 11,4, \mathrm{a} 11,3, \mathrm{~d} 11,0$

$\mathrm{H}, 7, \mathrm{r12}, 4, \mathrm{a} 12,3, \mathrm{~d} 12, \mathrm{O}$

$\mathrm{H}, 7, \mathrm{r13}, 4, \mathrm{a} 13,3, \mathrm{~d} 13, \mathrm{O}$

$\mathrm{H}, 5, \mathrm{r14}, 4, \mathrm{a} 14,3, \mathrm{~d} 14, \mathrm{O}$

$\mathrm{H}, 5, \mathrm{r} 15,4, \mathrm{a} 15,3, \mathrm{~d} 15,0$

$\mathrm{H}, 5, \mathrm{r} 16,4, \mathrm{a} 16,3, \mathrm{~d} 16,0)$

Variables:

$r 2=1.82185145$

$r 3=1.8050922$

a $3=99.97632493$

$r 4=1.42962155$

a $4=114.8021973$

$d 4=179.99994105$

$r 5=1.46381111$

a $5=114.11627738$

$d 5=66.34457402$

$r 6=1.19975575$

a $6=121.76406337$

$d 6=-0.00007572$

$r 7=1.46381121$

a $7=114.11627742$

$r 8=1.08734994$

a $8=110.05550208$

$\mathrm{d} 8=60.62557614$

$r 9=1.08734966$

a $9=110.05550143$

d $9=-60.62570516$

$r 10=1.08925904$

a $10=106.41881977$

d1 $0=179.99994023$

$r 11=1.0995436$

a $11=113.55281789$

$\mathrm{d} 11=74.22500716$

$r 12=1.09008656$

a12 $=109.66727724$

$\mathrm{d} 12=-47.64145952$

$r 13=1.09172007$

a13 $=108.25869454$

d13 $=-165.29029259$

$r 14=1.09008652$

a14 $=109.66728802$

d14 $=47.64142097$

$r 15=1.09954346$

a $15=113.55279566$

d1 $5=-74.22504968$

$r 16=1.09171994$

a $16=108.25870251$

d1 $6=165.2902838$

$d 7=-66.34440116$

B3LYP

$E=-683.630388$

$\# i=1$
$E=-686.120751$

$\# i=1$ 


\section{O-Methyl N,N-Dimethylthiocarbamate (3) GS HF/6-311+G(2d,p)}

C

$0,1, r 2$

C, $2, r 3,1$, a 3

$\mathrm{N}, 3, \mathrm{r} 4,2, \mathrm{a} 4,1, \mathrm{~d} 4,0$

$\mathrm{C}, 4, \mathrm{r} 5,3, \mathrm{a} 5,2, \mathrm{~d} 5,0$

$\mathrm{s}, 3, \mathrm{r} 6,2, \mathrm{a} 6,1, \mathrm{~d} 6,0$

$\mathrm{C}, 4, \mathrm{r} 7,3, \mathrm{a} 7,2, \mathrm{~d} 7,0$

$\mathrm{H}, 1, \mathrm{r} 8,2, \mathrm{a} 8,3, \mathrm{~d} 8,0$

$\mathrm{H}, 1, \mathrm{r} 9,2, \mathrm{a} 9,3, \mathrm{~d} 9, \mathrm{O}$

$\mathrm{H}, 1, \mathrm{r} 10,2, \mathrm{a} 10,3, \mathrm{~d} 10,0$

$\mathrm{H}, 7, \mathrm{r} 11,4, \mathrm{a} 11,3, \mathrm{~d} 11,0$

$\mathrm{H}, 7, \mathrm{r} 12,4, \mathrm{a} 12,3, \mathrm{~d} 12, \mathrm{O}$

$\mathrm{H}, 7, \mathrm{r13}, 4, \mathrm{a} 13,3, \mathrm{~d} 13,0$

$\mathrm{H}, 5, \mathrm{r} 14,4, \mathrm{a} 14,3, \mathrm{~d} 14, \mathrm{O}$

$\mathrm{H}, 5, \mathrm{r} 15,4, \mathrm{a} 15,3, \mathrm{~d} 15, \mathrm{O}$

$\mathrm{H}, 5, \mathrm{r} 16,4, \mathrm{a} 16,3, \mathrm{~d} 16,0$ Variables:

$r 2=1.41723341$

$r 3=1.3129619$

a $3=121.3785883$

$r 4=1.32536504$

a $4=110.7824084$

$\mathrm{d} 4=-179.99893011$

$r 5=1.45719881$

a $5=120.58356731$

$\mathrm{d} 5=-0.00482682$

$r 6=1.67679915$

a $6=123.10936082$

$d 6=-0.00354304$

$r 7=1.45058344$

a $7=123.04533312$

$d 7=179.98950641$

$r 8=1.07843123$

$a 8=110.54028155$

$\mathrm{d} 8=60.78688586$

$r 9=1.07843135$

a $9=110.54017386$

$d 9=-60.78601082$

$r 10=1.079094$

a $10=104.80439279$

d1 $0=-179.99955447$

$r 11=1.08437082$

$\mathrm{a} 11=109.66935996$

$\mathrm{d} 11=120.27828921$

$r 12=1.07360233$

a12 $=110.07061681$

$\mathrm{d} 12=-0.00999132$

$r 13=1.08437025$

a13 $=109.66814254$

d13 $=-120.29774415$

$r 14=1.08148627$

a14 $=111.2922408$

$\mathrm{d} 14=60.72075994$

$r 15=1.08147596$

a15 $=111.29174005$

d1 $5=-60.6764692$

$r 16=1.07956836$

a1 $6=107.95670075$

d1 $6=-179.97758811$
GS2

$0,1, \mathrm{~B} 1$

C, 2, B2, 1, A1

$\mathrm{N}, 3, \mathrm{~B} 3,2, \mathrm{~A} 2,1, \mathrm{D} 1,0$

$\mathrm{C}, 4, \mathrm{~B} 4,3, \mathrm{~A} 3,2, \mathrm{D} 2,0$

$\mathrm{S}, 3, \mathrm{~B} 5,2, \mathrm{~A} 4,1, \mathrm{D} 3,0$

$\mathrm{C}, 4, \mathrm{~B} 6,3, \mathrm{~A} 5,2, \mathrm{D} 4,0$

$\mathrm{H}, 1, \mathrm{~B} 7,2, \mathrm{~A} 6,3, \mathrm{D} 5,0$

$\mathrm{H}, 1, \mathrm{~B} 8,2, \mathrm{~A} 7,3, \mathrm{D} 6, \mathrm{O}$

$\mathrm{H}, 1, \mathrm{~B} 9,2, \mathrm{~A} 8,3, \mathrm{D} 7,0$

$\mathrm{H}, 7, \mathrm{~B} 10,4, \mathrm{~A} 9,3, \mathrm{D} 8, \mathrm{O}$

$\mathrm{H}, 7, \mathrm{~B} 11,4, \mathrm{~A} 10,3, \mathrm{D} 9, \mathrm{O}$

$\mathrm{H}, 7, \mathrm{~B} 12,4, \mathrm{~A} 11,3, \mathrm{D} 10,0$

$\mathrm{H}, 5, \mathrm{~B} 13,4, \mathrm{~A} 12,3, \mathrm{D} 11,0$

$\mathrm{H}, 5, \mathrm{~B} 14,4, \mathrm{~A} 13,3, \mathrm{D} 12,0$

$\mathrm{H}, 5, \mathrm{~B} 15,4, \mathrm{~A} 14,3, \mathrm{D} 13,0)$

Variables:

$\mathrm{B} 1=1.41693039$

$\mathrm{B} 2=1.3130116$

$\mathrm{B} 3=1.32509336$

$B 4=1.45483052$

$\mathrm{B} 5=1.67497507$

$B 6=1.45472475$

$\mathrm{B} 7=1.07852307$

$\mathrm{B} 8=1.07852214$

$\mathrm{B} 9=1.07916572$

$\mathrm{B} 10=1.08066081$

$\mathrm{B} 11=1.08061982$

$\mathrm{B} 12=1.08065934$

$B 13=1.08424792$

$B 14=1.08424829$

$\mathrm{B} 15=1.07340387$

$\mathrm{A} 1=121.11054422$

$\mathrm{A} 2=112.65658017$

$\mathrm{A} 3=123.64746254$

$\mathrm{A} 4=123.40195849$

$A 5=120.31789131$

$A 6=110.57135544$

$A 7=110.57043174$

$A 8=104.79542669$

$A 9=111.02568063$

$\mathrm{A} 10=107.90168524$

$\mathrm{A} 11=111.02441824$

$\mathrm{A} 12=109.65671283$

$\mathrm{A} 13=109.65728083$

$\mathrm{A} 14=110.98513136$

$\mathrm{D} 1=-179.97503888$

D2 $=-0.02117992$

D3 $=-0.02013689$

D $4=179.97463949$

$\mathrm{D} 5=60.81085991$

$\mathrm{D} 6=-60.8115856$

D7 $=179.99974406$

D $8=-60.35693008$

$\mathrm{D} 9=-179.99926311$

D1 $0=60.35875486$

$\mathrm{D} 11=-120.43912903$

$\mathrm{D} 12=120.43772191$

D13 $=-0.00094422$
$E=-683.634350$

$\# i=0$ 


\section{O-Methyl N,N-Dimethylthiocarbamate (3) GS B3LYP/6-311+G(2d,p)}

$\mathrm{C}$

$0,1, r 2$

$\mathrm{C}, 2, \mathrm{r3}, 1, \mathrm{a} 3$

$\mathrm{N}, 3, \mathrm{r} 4,2, \mathrm{a} 4,1, \mathrm{~d} 4,0$

$\mathrm{C}, 4, \mathrm{r} 5,3, \mathrm{a} 5,2, \mathrm{~d} 5,0$

$\mathrm{S}, 3, \mathrm{r} 6,2, \mathrm{a} 6,1, \mathrm{~d} 6,0$

$\mathrm{C}, 4, \mathrm{r} 7,3, \mathrm{a} 7,2, \mathrm{~d} 7,0$

$\mathrm{H}, 1, \mathrm{r} 8,2, \mathrm{a} 8,3, \mathrm{~d} 8,0$

$\mathrm{H}, 1, \mathrm{r} 9,2, \mathrm{a} 9,3, \mathrm{~d} 9, \mathrm{O}$

$\mathrm{H}, 1, \mathrm{r} 10,2, \mathrm{a} 10,3, \mathrm{~d} 10,0$

$\mathrm{H}, 7, \mathrm{r} 11,4, \mathrm{a} 11,3, \mathrm{~d} 11,0$

$\mathrm{H}, 7, \mathrm{r12}, 4, \mathrm{a} 12,3, \mathrm{~d} 12, \mathrm{O}$

$\mathrm{H}, 7, \mathrm{r13}, 4, \mathrm{a} 13,3, \mathrm{~d} 13, \mathrm{O}$

$\mathrm{H}, 5, \mathrm{r} 14,4, \mathrm{a} 14,3, \mathrm{~d} 14, \mathrm{O}$

$\mathrm{H}, 5, \mathrm{r} 15,4, \mathrm{a} 15,3, \mathrm{~d} 15,0$

$\mathrm{H}, 5, \mathrm{r} 16,4, \mathrm{a} 16,3, \mathrm{~d} 16,0$ Variables:

$r 2=1.43407912$

$r 3=1.34842907$

a $3=119.10763021$

$r 4=1.35022301$

$a_{4}=110.10278602$

$\mathrm{d} 4=-177.95348874$

$r 5=1.46248303$

a $5=121.34598134$

$d 5=-2.85051529$

$r 6=1.67233748$

a $6=123.67315485$

$d 6=1.95115131$

$r 7=1.45525184$

a $7=121.83537637$

$d 7=-174.38585992$

$r 8=1.08910816$

$a 8=110.61656637$

$\mathrm{d} 8=60.53322257$

$r 9=1.08899129$

a $9=110.54475159$

d $9=-60.58474605$

$r 10=1.08819417$

a $10=104.66301888$

$\mathrm{d} 10=179.97381407$

$r 11=1.09497027$

$\mathrm{a} 11=110.48756089$

$\mathrm{d} 11=108.65246516$

$r 12=1.08523101$

a12=109.29199213

d12 $=-11.42522954$

$r 13=1.09247158$

a $13=109.56062716$

d13 $=-131.56273165$

$r 14=1.08724759$

a $14=110.8703952$

d1 $4=44.67150826$

$r 15=1.09445125$

a $15=111.54509806$

$\mathrm{d} 15=-76.38509104$

$r 16=1.08899832$

a $16=108.19056823$

d16=164.1307192

$E=-686.1240648$

$\# i=0$ 


\section{O-Methyl N,N-Dimethylthiocarbamate (3) TS1 6-311+G(2d,p)}

C

$0,1, r 2$

$\mathrm{C}, 2, \mathrm{r3}, 1, \mathrm{a} 3$

$\mathrm{N}, 3, \mathrm{r} 4,2, \mathrm{a} 4,1, \mathrm{~d} 4,0$

$\mathrm{C}, 4, \mathrm{r} 5,3, \mathrm{a} 5,2, \mathrm{~d} 5,0$

$\mathrm{s}, 3, \mathrm{r} 6,2, \mathrm{a} 6,1, \mathrm{~d} 6,0$

$\mathrm{C}, 4, \mathrm{r} 7,3, \mathrm{a} 7,2, \mathrm{~d} 7,0$

$\mathrm{H}, 1, \mathrm{r} 8,2, \mathrm{a} 8,3, \mathrm{~d} 8,0$

$\mathrm{H}, 1, \mathrm{r} 9,2, \mathrm{a} 9,3, \mathrm{~d} 9,0$

$\mathrm{H}, 1, \mathrm{r10}, 2, \mathrm{a} 10,3, \mathrm{~d} 10,0$

$\mathrm{H}, 7, \mathrm{r} 11,4, \mathrm{a} 11,3, \mathrm{~d} 11, \mathrm{O}$

$\mathrm{H}, 7, \mathrm{r} 12,4, \mathrm{a} 12,3, \mathrm{~d} 12, \mathrm{O}$

$\mathrm{H}, 7, \mathrm{r13}, 4, \mathrm{a} 13,3, \mathrm{~d} 13, \mathrm{O}$

$\mathrm{H}, 5, \mathrm{r} 14,4, \mathrm{a} 14,3, \mathrm{~d} 14,0$

$\mathrm{H}, 5, \mathrm{r} 15,4, \mathrm{a} 15,3, \mathrm{~d} 15,0$

$\mathrm{H}, 5, \mathrm{r} 16,4, \mathrm{a} 16,3, \mathrm{~d} 16,0 \mathrm{~J}$

Variables:

$r 2=1.41650606$

$r 3=1.29547756$

a $3=121.1177767$

$r 4=1.40893818$

$a_{4}=109.88024118$

$d 4=-179.99490331$

$r 5=1.4564693$

a $5=112.88043333$

$\mathrm{d} 5=-115.49803032$

$r 6=1.63908081$

a $6=125.10379028$

$d 6=0.00021287$

$r 7=1.45648093$

a $7=112.87514963$

$d 7=115.43194322$

$r 8=1.07964216$

$a 8=110.35154602$

$\mathrm{d} 8=-60.58920878$

$r 9=1.07821554$

a $9=105.10463979$

d $9=-179.99751775$

$r 10=1.07964396$

a $10=110.35331101$

$\mathrm{d} 10=60.59355332$

$r 11=1.08713271$

$\mathrm{a} 11=112.62713442$

$\mathrm{d} 11=69.60353036$

$r 12=1.08220606$

a $12=109.8273211$

$\mathrm{d} 12=-51.89856101$

$r 13=1.08241382$

a13 $=108.08660727$

$\mathrm{d} 13=-169.61596856$

$r 14=1.0822042$

a1 $4=109.82891629$

$\mathrm{d} 14=51.88552086$

$r 15=1.08713187$

a1 $5=112.62943438$

$\mathrm{d} 15=-69.61708159$

$r 16=1.08241338$

a1 $6=108.08451055$

d1 $6=169.60402794$

\section{$E=-683.608483$ \\ $\# i=1$}

C

$0,1, r 2$

C, $2, r 3,1$, a 3

$\mathrm{N}, 3, \mathrm{r} 4,2, \mathrm{a} 4,1, \mathrm{~d} 4,0$

C, 4, r5, 3, a 5, 2, d5, 0

$\mathrm{s}, 3, \mathrm{r} 6,2, \mathrm{a} 6,1, \mathrm{~d} 6,0$

$\mathrm{C}, 4, \mathrm{r} 7,3, \mathrm{a} 7,2, \mathrm{~d} 7,0$

$\mathrm{H}, 1, \mathrm{r} 8,2, \mathrm{a} 8,3, \mathrm{~d} 8,0$

$\mathrm{H}, 1, \mathrm{rg}, 2, \mathrm{a} 9,3, \mathrm{~d} 9, \mathrm{O}$

$\mathrm{H}, 1, \mathrm{r} 10,2, \mathrm{a} 10,3, \mathrm{~d} 10,0$

$\mathrm{H}, 7, \mathrm{r} 11,4, \mathrm{a} 11,3, \mathrm{~d} 11,0$

$\mathrm{H}, 7, \mathrm{r} 12,4, \mathrm{a} 12,3, \mathrm{~d} 12,0$

$\mathrm{H}, 7, \mathrm{r13}, 4, \mathrm{a} 13,3, \mathrm{~d} 13, \mathrm{O}$

$\mathrm{H}, 5, \mathrm{r14}, 4, \mathrm{a} 14,3, \mathrm{~d} 14, \mathrm{O}$

$\mathrm{H}, 5, \mathrm{r15}, 4, \mathrm{a} 15,3, \mathrm{~d} 15,0$

$\mathrm{H}, 5, \mathrm{r16}, 4, \mathrm{a} 16,3, \mathrm{~d} 16,0)$

Variables:

$r 2=1.43705206$

$r 3=1.32929611$

a $3=119.13331339$

$r 4=1.41977459$

$a_{4}=109.22250733$

$\mathrm{d} 4=-179.99642592$

$r 5=1.46655418$

a $5=112.65184335$

$d 5=-115.9285187$

$r 6=1.64777567$

a $6=125.05639695$

$d 6=0.00687914$

$r 7=1.4665519$

a $7=112.65196802$

$\mathrm{d} 7=115.98000136$

$r 8=1.08979309$

a $8=110.22072221$

$\mathrm{d} 8=-60.29675105$

$r 9=1.08718756$

a $9=104.96205086$

$d 9=179.9682574$

$r 10=1.08977886$

a $10=110.22209031$

d1 $0=60.22726275$

$r 11=1.09841132$

a $11=112.56089376$

$\mathrm{d} 11=68.0010378$

$r 12=1.09023312$

a12=109.72064536

$\mathrm{d} 12=-53.41468521$

$r 13=1.09114185$

a13=108.19595731

d13 $=-171.26399017$

$r 14=1.09023132$

a $14=109.72056255$

d1 $4=53.41369299$

$r 15=1.09841748$

a $15=112.5606645$

$\mathrm{d} 15=-68.00151056$

$r 16=1.09114578$

a $16=108.19980019$

d1 $6=171.26296377$
B3LYP

$E=-686.099160$
$\# i=1$ 


\section{O-Methyl N,N-Dimethylthiocarbamate (3) TS2 6-311+G(2d,p)}

C

$0,1, r 2$

C, $2, r 3,1$, a 3

$\mathrm{N}, 3, \mathrm{r} 4,2, \mathrm{a} 4,1, \mathrm{~d} 4,0$

$\mathrm{C}, 4, \mathrm{r} 5,3, \mathrm{a} 5,2, \mathrm{~d} 5,0$

$\mathrm{s}, 3, \mathrm{r} 6,2, \mathrm{a} 6,1, \mathrm{~d} 6,0$

$\mathrm{C}, 4, \mathrm{r} 7,3, \mathrm{a} 7,2, \mathrm{~d} 7,0$

$\mathrm{H}, 1, \mathrm{r} 8,2, \mathrm{a} 8,3, \mathrm{~d} 8,0$

$\mathrm{H}, 1, \mathrm{r} 9,2, \mathrm{a} 9,3, \mathrm{~d} 9,0$

$\mathrm{H}, 1, \mathrm{r} 10,2, \mathrm{a} 10,3, \mathrm{~d} 10,0$

$\mathrm{H}, 7, \mathrm{r} 11,4, \mathrm{a} 11,3, \mathrm{~d} 11,0$

$\mathrm{H}, 7, \mathrm{r} 12,4, \mathrm{a} 12,3, \mathrm{~d} 12,0$

$\mathrm{H}, 7, \mathrm{r13}, 4, \mathrm{a} 13,3, \mathrm{~d} 13, \mathrm{O}$

$\mathrm{H}, 5, \mathrm{r} 14,4, \mathrm{a} 14,3, \mathrm{~d} 14, \mathrm{O}$

$\mathrm{H}, 5, \mathrm{r} 15,4, \mathrm{a} 15,3, \mathrm{~d} 15,0$

$\mathrm{H}, 5, \mathrm{r16}, 4, \mathrm{a} 16,3, \mathrm{~d} 16,0$

Variables:

$r 2=1.41602832$

$r 3=1.30538593$

a $3=121.34225653$

$r 4=1.40684079$

$a_{4}=111.9452138$

$d 4=179.99981866$

$r 5=1.45623398$

a $5=112.64035765$

$d 5=64.70291712$

$r 6=1.62848585$

a $6=125.2825361$

$d 6=-0.00020597$

$r 7=1.45623114$

a $7=112.64284743$

$d 7=-64.67879333$

$r 8=1.07959983$

a $8=110.30314905$

$d 8=60.54302547$

$r 9=1.07960044$

a $9=110.30318434$

$\mathrm{d} 9=-60.54317063$

$r 10=1.07863384$

a $10=105.28014996$

$\mathrm{d} 10=180$.

$r 11=1.08848143$

$\mathrm{a} 11=113.08154765$

$\mathrm{d} 11=69.59116571$

$r 12=1.0823573$

a12 $=109.50974827$

$\mathrm{d} 12=-52.20073874$

$r 13=1.08215912$

a $13=108.2084426$

d13 $=-169.75314375$

$r 14=1.08235516$

a1 $4=109.51011337$

d1 $4=52.14527068$

$r 15=1.08848493$

a $15=113.08016289$

$\mathrm{d} 15=-69.64468287$

$r 16=1.08215935$

a $16=108.2092768$

d1 $6=169.69854767$
$E=-683.606743$

$\# i=1$
$\mathrm{C}$

$0,1, r 2$

C, $2, \mathrm{r3}, 1, \mathrm{a} 3$

$\mathrm{N}, 3, \mathrm{r} 4,2, \mathrm{a} 4,1, \mathrm{~d} 4,0$

C, 4, r5, 3, a 5, 2, d5, 0

$\mathrm{s}, 3, \mathrm{r} 6,2, \mathrm{a} 6,1, \mathrm{~d} 6,0$

$\mathrm{C}, 4, \mathrm{r} 7,3, \mathrm{a} 7,2, \mathrm{~d} 7,0$

$\mathrm{H}, 1, \mathrm{r} 8,2, \mathrm{a} 8,3, \mathrm{~d} 8,0$

$\mathrm{H}, 1, \mathrm{rg}, 2, \mathrm{a} 9,3, \mathrm{~d} 9, \mathrm{O}$

$\mathrm{H}, 1, \mathrm{r10}, 2, \mathrm{a} 10,3, \mathrm{~d} 10,0$

$\mathrm{H}, 7, \mathrm{r} 11,4, \mathrm{a} 11,3, \mathrm{~d} 11,0$

$\mathrm{H}, 7, \mathrm{r} 12,4, \mathrm{a} 12,3, \mathrm{~d} 12,0$

$\mathrm{H}, 7, \mathrm{r13}, 4, \mathrm{a} 13,3, \mathrm{~d} 13, \mathrm{O}$

$\mathrm{H}, 5, \mathrm{r} 14,4, \mathrm{a} 14,3, \mathrm{~d} 14, \mathrm{O}$

$\mathrm{H}, 5, \mathrm{r} 15,4, \mathrm{a} 15,3, \mathrm{~d} 15,0$

$\mathrm{H}, 5, \mathrm{r} 16,4, \mathrm{a} 16,3, \mathrm{~d} 16,0)$

Variables:

$r 2=1.43600252$

$r 3=1.33904304$

a $3=119.68068498$

$r 4=1.41637507$

a $4=111.4583668$

$d 4=179.98829748$

$r 5=1.46577845$

a $5=113.15179794$

$d 5=65.10764467$

$r 6=1.63888171$

a $6=124.99611001$

$d 6=-0.01257148$

$r 7=1.46576989$

a $7=113.15109599$

$d 7=-65.0962294$

$r 8=1.08981053$

a $8=110.17295091$

$\mathrm{d} 8=60.24746955$

$r 9=1.08980481$

a $9=110.1743346$

$d 9=-60.17341927$

r $10=1.08769934$

a $10=105.19060792$

d1 $0=-179.9649841$

$r 11=1.09909157$

a11 $=113.28219515$

$d 11=69.99438027$

$r 12=1.0906379$

a12 $=109.36807997$

$\mathrm{d} 12=-51.79226061$

$r 13=1.09126034$

a $13=108.25195372$

d13 $=-169.36062551$

$r 14=1.09063509$

a $14=109.36709827$

d1 $4=51.72268335$

$r 15=1.09909913$

a1 $5=113.28219365$

d15 $=-70.05895941$

$r 16=1.09126158$

a $16=108.2543316$

d1 $6=169.29233907$
B3LYP

\author{
$E=-686.096694$ \\ $\# i=1$
}




\section{Methyl N,N-Dimethyldithiocarbamate (4) GS HF/6-311+G(2d,p)}

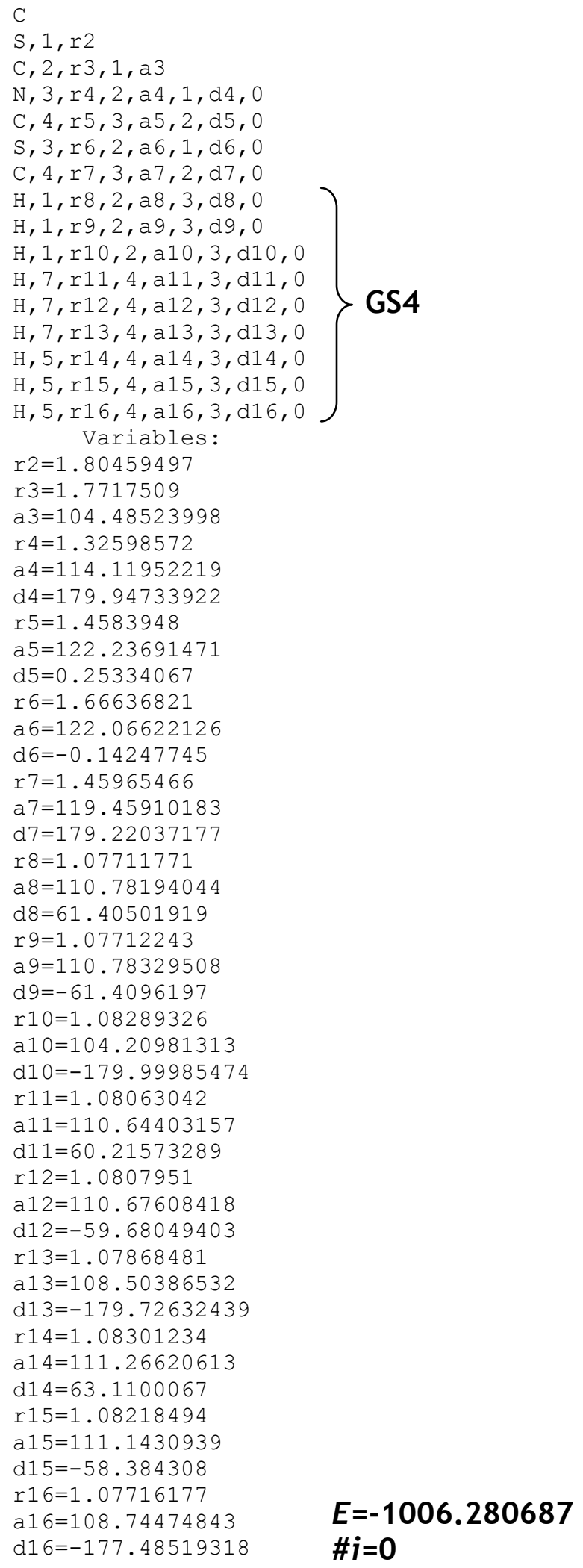


C

$\mathrm{S}, 1, \mathrm{r} 2$

C, $2, r 3,1$, a 3

$\mathrm{N}, 3, \mathrm{r} 4,2, \mathrm{a} 4,1, \mathrm{~d} 4,0$

$\mathrm{C}, 4, \mathrm{r} 5,3, \mathrm{a} 5,2, \mathrm{~d} 5,0$

$\mathrm{s}, 3, \mathrm{r} 6,2, \mathrm{a} 6,1, \mathrm{~d} 6,0$

$\mathrm{C}, 4, \mathrm{r} 7,3, \mathrm{a} 7,2, \mathrm{~d} 7,0$

$\mathrm{H}, 1, \mathrm{r} 8,2, \mathrm{a} 8,3, \mathrm{~d} 8,0$

$\mathrm{H}, 1, \mathrm{rg}, 2, \mathrm{a} 9,3, \mathrm{~d} 9, \mathrm{O}$

$\mathrm{H}, 1, \mathrm{r} 10,2, \mathrm{a} 10,3, \mathrm{~d} 10,0$

$\mathrm{H}, 7, \mathrm{r} 11,4, \mathrm{a} 11,3, \mathrm{~d} 11, \mathrm{O}$

$\mathrm{H}, 7, \mathrm{r} 12,4, \mathrm{a} 12,3, \mathrm{~d} 12,0$

GS2

$\mathrm{H}, 7, \mathrm{r13}, 4, \mathrm{a} 13,3, \mathrm{~d} 13,0$

$\mathrm{H}, 5, \mathrm{r} 14,4, \mathrm{a} 14,3, \mathrm{~d} 14,0$

$\mathrm{H}, 5, \mathrm{r} 15,4, \mathrm{a} 15,3, \mathrm{~d} 15,0$

$\mathrm{H}, 5, \mathrm{r16}, 4, \mathrm{a} 16,3, \mathrm{~d} 16,0$

Variables:

$r 2=1.81298714$

$r 3=1.79435744$

a $3=102.94175316$

$r 4=1.35181215$

a $4=112.58382342$

$\mathrm{d} 4=-178.6053091$

$r 5=1.46160514$

a $5=122.40621796$

$d 5=-2.74616592$

$r 6=1.66820125$

a $6=122.12989868$

$d 6=1.60321642$

$r 7=1.46032445$

a $7=121.64949958$

$\mathrm{d} 7=-175.88393775$

$r 8=1.08690892$

$a 8=110.65385668$

$d 8=61.50781391$

$r 9=1.08691651$

a $9=110.5536815$

$\mathrm{d} 9=-60.63122407$

$r 10=1.09105922$

a1 $0=104.52329482$

d1 $0=-179.56778124$

$r 11=1.09465907$

$\mathrm{a} 11=110.31592117$

$\mathrm{d} 11=107.84142354$

$r 12=1.08491418$

a12 $=109.32182257$

$\mathrm{d} 12=-12.18255323$

$r 13=1.09203648$

a13 $=109.32747775$

$\mathrm{d} 13=-132.42984797$

$r 14=1.09092449$

a1 $4=111.02516217$

d14 $=51.82520487$

$r 15=1.0940429$

a15 $=111.60343134$

$\mathrm{d} 15=-69.69104519$

$r 16=1.08799373$

a1 $6=108.44612407$

d1 $6=170.67563652$

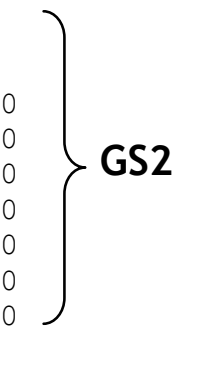

C

$\mathrm{S}, 1, \mathrm{~B} 1$

$\mathrm{C}, 2, \mathrm{~B} 2,1, \mathrm{~A} 1$

$\mathrm{N}, 3, \mathrm{~B} 3,2, \mathrm{~A} 2,1, \mathrm{D} 1,0$

$C, 4, B 4,3, A 3,2, D 2,0$

$\mathrm{S}, 3, \mathrm{~B} 5,2, \mathrm{~A} 4,1, \mathrm{D} 3,0$

$\mathrm{C}, 4, \mathrm{~B} 6,3, \mathrm{~A} 5,2, \mathrm{D} 4,0$

$\mathrm{H}, 1, \mathrm{~B} 7,2, \mathrm{~A} 6,3, \mathrm{D} 5,0$

$\mathrm{H}, 1, \mathrm{~B} 8,2, \mathrm{~A} 7,3, \mathrm{D} 6, \mathrm{O}$

$\mathrm{H}, 1, \mathrm{~B} 9,2, \mathrm{~A} 8,3, \mathrm{D} 7,0$

$\mathrm{H}, 7, \mathrm{~B} 10,4, \mathrm{~A} 9,3, \mathrm{D} 8, \mathrm{O}$

$\mathrm{H}, 7, \mathrm{~B} 11,4, \mathrm{~A} 10,3, \mathrm{D} 9, \mathrm{O}$

$\mathrm{H}, 7, \mathrm{~B} 12,4, \mathrm{~A} 11,3, \mathrm{D} 10,0$

$\mathrm{H}, 5, \mathrm{~B} 13,4, \mathrm{~A} 12,3, \mathrm{D} 11, \mathrm{O}$

$\mathrm{H}, 5, \mathrm{~B} 14,4, \mathrm{~A} 13,3, \mathrm{D} 12,0$

$\mathrm{H}, 5, \mathrm{~B} 15,4, \mathrm{~A} 14,3, \mathrm{D} 13,0$ Variables:

$\mathrm{B} 1=1.81315092$

$\mathrm{B} 2=1.79349592$

$\mathrm{B} 3=1.34992783$

$\mathrm{B} 4=1.46245697$

$\mathrm{B} 5=1.66655147$

$\mathrm{B} 6=1.46326645$

$\mathrm{B} 7=1.08700626$

$\mathrm{B} 8=1.08701133$

$\mathrm{B} 9=1.09092323$

$\mathrm{B} 10=1.0919403$

$\mathrm{B} 11=1.08791326$

$\mathrm{B} 12=1.09043817$

$\mathrm{B} 13=1.08684915$

$B 14=1.09408555$

$B 15=1.0914268$

$\mathrm{A} 1=102.66259456$

$\mathrm{A} 2=113.20633888$

$\mathrm{A} 3=122.1675183$

$\mathrm{A} 4=122.96834698$

$\mathrm{A} 5=119.19439759$

$A 6=110.53659137$

$\mathrm{A} 7=110.61405981$

$A 8=104.62917679$

$A 9=110.85013154$

$\mathrm{A} 10=108.58531072$

$\mathrm{A} 11=110.48022542$

$\mathrm{A} 12=108.8633787$

A13 $=111.58744277$

$\mathrm{A} 14=110.81856645$

$\mathrm{D} 1=179.08724443$

D2 $=1.57557952$

D3 $=-1.28966288$

D $4=177.19111009$

$\mathrm{D} 5=60.69839802$

D $6=-61.36041559$

$\mathrm{D} 7=179.67424938$

D $8=-61.09979239$

$\mathrm{D} 9=178.47031782$

$\mathrm{D} 10=58.10560709$

D1 $1=-170.67794149$

$\mathrm{D} 12=69.31388276$

D13 $=-51.77994341$
$E=-1009.096894$ $\# i=0$
$E=-1009.097170$

$\# i=0$ 


\section{Methyl N,N-Dimethyldithiocarbamate (4) TS1 6-311+G(2d,p)}

C

$\mathrm{S}, 1, \mathrm{r} 2$

$\mathrm{C}, 2, \mathrm{r} 3,1, \mathrm{a} 3$

$\mathrm{S}, 3, \mathrm{r} 4,2, \mathrm{a} 4,1, \mathrm{~d} 4,0$

$\mathrm{N}, 3, \mathrm{r} 5,2, \mathrm{a} 5,1, \mathrm{~d} 5,0$

$c, 5, r 6,3, a 6,2, d 6,0$

$\mathrm{C}, 5, \mathrm{r} 7,3, \mathrm{a} 7,2, \mathrm{~d} 7,0$

$\mathrm{H}, 1, \mathrm{r} 8,2, \mathrm{a} 8,3, \mathrm{~d} 8,0$

$\mathrm{H}, 1, \mathrm{r} 9,2, \mathrm{a} 9,3, \mathrm{~d} 9, \mathrm{O}$

$\mathrm{H}, 1, \mathrm{r} 10,2, \mathrm{a} 10,3, \mathrm{~d} 10,0$

$\mathrm{H}, 6, \mathrm{r} 11,5, \mathrm{a} 11,3, \mathrm{~d} 11, \mathrm{O}$

$\mathrm{H}, 6, \mathrm{r} 12,5, \mathrm{a} 12,3, \mathrm{~d} 12, \mathrm{O}$

$\mathrm{H}, 6, \mathrm{r} 13,5, \mathrm{a} 13,3, \mathrm{~d} 13,0$

$\mathrm{H}, 7, \mathrm{r} 14,5, \mathrm{a} 14,3, \mathrm{~d} 14,0$

$\mathrm{H}, 7, \mathrm{r} 15,5, \mathrm{a} 15,3, \mathrm{~d} 15,0$

$\mathrm{H}, 7, \mathrm{r} 16,5, \mathrm{a} 16,3, \mathrm{~d} 16,0$

$r 2=1.80401675$

$r 3=1.73108639$

a $3=105.03598243$

$r 4=1.62370723$

a $4=126.38470778$

$\mathrm{d} 4=0.00762008$

$r 5=1.41859447$

a $5=108.01905669$

$d 5=-179.99519879$

$r 6=1.45729912$

a $6=112.87214624$

$d 6=115.37860539$

$r 7=1.45729182$

a $7=112.87242423$

$r 8=1.07899361$

a $8=110.63170521$

$\mathrm{d} 8=61.04569863$

$r 9=1.07899592$

a $9=110.63207187$

$\mathrm{d} 9=-61.07290093$

$r 10=1.08221604$

a $10=105.53548181$

$\mathrm{d} 10=179.98641963$

$r 11=1.08699885$

a11 $=112.54069145$

$\mathrm{d} 11=69.0586485$

$r 12=1.08199083$

a12=109.68448839

$\mathrm{d} 12=-52.32404185$

$r 13=1.08241818$

a13 $=108.14283521$

d13 $=-170.18340718$

$r 14=1.08199109$

a14=109.68499757

d1 $4=52.31440142$

r $15=1.08700177$

a $15=112.54077281$

d1 $5=-69.0669537$

$r 16=1.08241905$

a $16=108.14372045$

d1 $6=170.17448343$

$d 7=-115.37779728$
C

$\mathrm{S}, 1, \mathrm{r} 2$

$\mathrm{C}, 2, \mathrm{r} 3,1, \mathrm{a} 3$

$\mathrm{S}, 3, \mathrm{r} 4,2, \mathrm{a} 4,1, \mathrm{~d} 4,0$

$\mathrm{N}, 3, \mathrm{r} 5,2, \mathrm{a} 5,1, \mathrm{~d} 5,0$

$c, 5, r 6,3, a 6,2, d 6,0$

C, 5, r7 $, 3, a 7,2, d 7,0$

$\mathrm{H}, 1, \mathrm{r} 8,2, \mathrm{a} 8,3, \mathrm{~d} 8,0$

$\mathrm{H}, 1, \mathrm{r} 9,2, \mathrm{a} 9,3, \mathrm{~d} 9,0$

$\mathrm{H}, 1, \mathrm{r} 10,2, \mathrm{a} 10,3, \mathrm{~d} 10,0$

$\mathrm{H}, 6, \mathrm{r} 11,5, \mathrm{a} 11,3, \mathrm{~d} 11,0$

$\mathrm{H}, 6, \mathrm{r} 12,5, \mathrm{a} 12,3, \mathrm{~d} 12, \mathrm{O}$

$\mathrm{H}, 6, \mathrm{r13}, 5, \mathrm{a} 13,3, \mathrm{~d} 13, \mathrm{O}$

$\mathrm{H}, 7, \mathrm{r} 14,5, \mathrm{a} 14,3, \mathrm{~d} 14,0$

$\mathrm{H}, 7, \mathrm{r} 15,5, \mathrm{a} 15,3, \mathrm{~d} 15,0$

$\mathrm{H}, 7, \mathrm{r16}, 5, \mathrm{a} 16,3, \mathrm{~d} 16,0$

$r 2=1.81656$

$r 3=1.74034$

a $3=104.10567$

$r 4=1.64227$

a $4=126.72234$

$\mathrm{d} 4=-0.00642$

$r 5=1.43098$

a $5=107.18373$

d5 $=179.99231$

$r 6=1.46721$

a $6=112.72407$

d $6=115.74271$

$r 7=1.46722$

a $7=112.72017$

$r 8=1.08848$

a $8=110.20351$

$\mathrm{d} 8=60.49508$

$r 9=1.08848$

a $9=110.20746$

$\mathrm{d} 9=-60.59074$

$r 10=1.09003$

a1 $0=106.03636$

$\mathrm{d} 10=179.95236$

$r 11=1.09785$

a $11=112.51683$

$\mathrm{d} 11=67.69644$

$r 12=1.09024$

a $12=109.54314$

$\mathrm{d} 12=-53.51458$

$r 13=1.09118$

a $13=108.2948$

d13 $=-171.51623$

$r 14=1.09024$

a1 $4=109.54367$

d1 $4=53.49276$

$r 15=1.09785$

a $15=112.51461$

d1 $5=-67.71655$

$r 16=1.09118$

a $16=108.29472$

d1 $6=171.49614$

$d 7=-115.69505$
B3LYP

$E=-1009.077065$

$\# i=1$ 


\section{Methyl N,N-Dimethyldithiocarbamate (4) TS2 6-311+G(2d,p)}

C

$\mathrm{S}, 1, \mathrm{r} 2$

C, $2, r 3,1$, a 3

$\mathrm{N}, 3, \mathrm{r} 4,2, \mathrm{a} 4,1, \mathrm{~d} 4,0$

$\mathrm{C}, 4, \mathrm{r} 5,3, \mathrm{a} 5,2, \mathrm{~d} 5,0$

$\mathrm{S}, 3, \mathrm{r} 6,2, \mathrm{a} 6,1, \mathrm{~d} 6,0$

$\mathrm{C}, 4, \mathrm{r} 7,3, \mathrm{a} 7,2, \mathrm{~d} 7,0$

$\mathrm{H}, 1, \mathrm{r} 8,2, \mathrm{a} 8,3, \mathrm{~d} 8,0$

$\mathrm{H}, 1, \mathrm{r9}, 2, \mathrm{a} 9,3, \mathrm{~d} 9,0$

$\mathrm{H}, 1, \mathrm{r} 10,2, \mathrm{a} 10,3, \mathrm{~d} 10,0$

$\mathrm{H}, 7, \mathrm{r} 11,4, \mathrm{a} 11,3, \mathrm{~d} 11,0$

$\mathrm{H}, 7, \mathrm{r} 12,4, \mathrm{a} 12,3, \mathrm{~d} 12,0$

$\mathrm{H}, 7, \mathrm{r13}, 4, \mathrm{a} 13,3, \mathrm{~d} 13, \mathrm{O}$

$\mathrm{H}, 5, \mathrm{r} 14,4, \mathrm{a} 14,3, \mathrm{~d} 14, \mathrm{O}$

$\mathrm{H}, 5, \mathrm{r} 15,4, \mathrm{a} 15,3, \mathrm{~d} 15,0$

$\mathrm{H}, 5, \mathrm{r16}, 4, \mathrm{a} 16,3, \mathrm{~d} 16,0$

$r 2=1.80427004$

$r 3=1.75383487$

a $3=105.23503451$

$r 4=1.41409831$

a $4=113.44892155$

$\mathrm{d} 4=-179.99809534$

$r 5=1.45426821$

a $5=114.78813003$

$d 5=67.53256687$

$r 6=1.6164598$

a $6=124.71665469$

$d 6=0.00178773$

$r 7=1.45426837$

a $7=114.7885472$

$r 8=1.07842322$

a $8=110.5259788$

$d 8=61.06227056$

$r 9=1.07842294$

a $9=110.52608552$

d $9=-61.06353486$

$r 10=1.08213292$

a $10=105.30447918$

$\mathrm{d} 10=179.99933778$

$r 11=1.08901628$

$\mathrm{a} 11=113.49189731$

$\mathrm{d} 11=74.80664446$

$r 12=1.08161514$

a $12=109.57244248$

$\mathrm{d} 12=-47.01217196$

$r 13=1.08258962$

a13 $=108.13011617$

d13 $=-164.6679758$

$r 14=1.08161528$

a14 $=109.57242917$

$\mathrm{d} 14=47.01267054$

$r 15=1.0890161$

a $15=113.49182973$

d1 $5=-74.80617241$

$r 16=1.08258959$

a $16=108.13014148$

$\mathrm{d} 16=164.66839128$

$d 7=-67.52735011$
$E=-1006.254111$ $\# i=1$
C

$\mathrm{S}, 1, \mathrm{r} 2$

C, 2, r3, 1, a 3

$\mathrm{N}, 3, \mathrm{r} 4,2, \mathrm{a} 4,1, \mathrm{~d} 4,0$

C, 4, r5 $, 3, a 5,2, d 5,0$

$\mathrm{s}, 3, \mathrm{r} 6,2, \mathrm{a} 6,1, \mathrm{~d} 6,0$

$\mathrm{C}, 4, \mathrm{r} 7,3, \mathrm{a} 7,2, \mathrm{~d} 7,0$

$\mathrm{H}, 1, \mathrm{r} 8,2, \mathrm{a} 8,3, \mathrm{~d} 8,0$

$\mathrm{H}, 1, \mathrm{rg}, 2, \mathrm{a} 9,3, \mathrm{~d} 9, \mathrm{O}$

$\mathrm{H}, 1, \mathrm{r} 10,2, \mathrm{a} 10,3, \mathrm{~d} 10,0$

$\mathrm{H}, 7, \mathrm{r} 11,4, \mathrm{a} 11,3, \mathrm{~d} 11,0$

$\mathrm{H}, 7, \mathrm{r12}, 4, \mathrm{a} 12,3, \mathrm{~d} 12, \mathrm{O}$

$\mathrm{H}, 7, \mathrm{r13}, 4, \mathrm{a} 13,3, \mathrm{~d} 13,0$

$\mathrm{H}, 5, \mathrm{r14}, 4, \mathrm{a} 14,3, \mathrm{~d} 14, \mathrm{O}$

$\mathrm{H}, 5, \mathrm{r} 15,4, \mathrm{a} 15,3, \mathrm{~d} 15, \mathrm{O}$

$\mathrm{H}, 5, \mathrm{r16}, 4, \mathrm{a} 16,3, \mathrm{~d} 16,0$ J

$r 2=1.81464422$

$r 3=1.76804896$

a $3=104.46857446$

$r 4=1.42207261$

a $4=112.93399471$

$\mathrm{d} 4=-179.98930573$

$r 5=1.46280304$

a $5=115.14608268$

$d 5=67.93983546$

$r 6=1.63615105$

a $6=124.56485718$

$d 6=0.01185224$

$r 7=1.4628049$

a $7=115.14381215$

$r 8=1.08804073$

$a 8=110.11801456$

$\mathrm{d} 8=-60.56029966$

$r 9=1.09023872$

a $9=105.90412042$

$d 9=179.9541057$

$r 10=1.08803935$

a $10=110.11538574$

$\mathrm{d} 10=60.46853339$

$r 11=1.09989449$

a $11=113.65608042$

$\mathrm{d} 11=74.83453495$

$r 12=1.0900158$

a $12=109.41715967$

$\mathrm{d} 12=-46.89952064$

$r 13=1.09179093$

a13=108.2953875

d13 $=-164.59516161$

$r 14=1.09001738$

a1 $4=109.41722127$

d1 $4=46.91608254$

$r 15=1.09989147$

a $15=113.65829428$

d1 $5=-74.82031749$

$r 16=1.09178997$

a1 $6=108.29314433$

d1 $6=164.61051286$

$d 7=-67.9529511$
B3LYP

\section{$E=-1009.070343$}

$\# i=1$ 

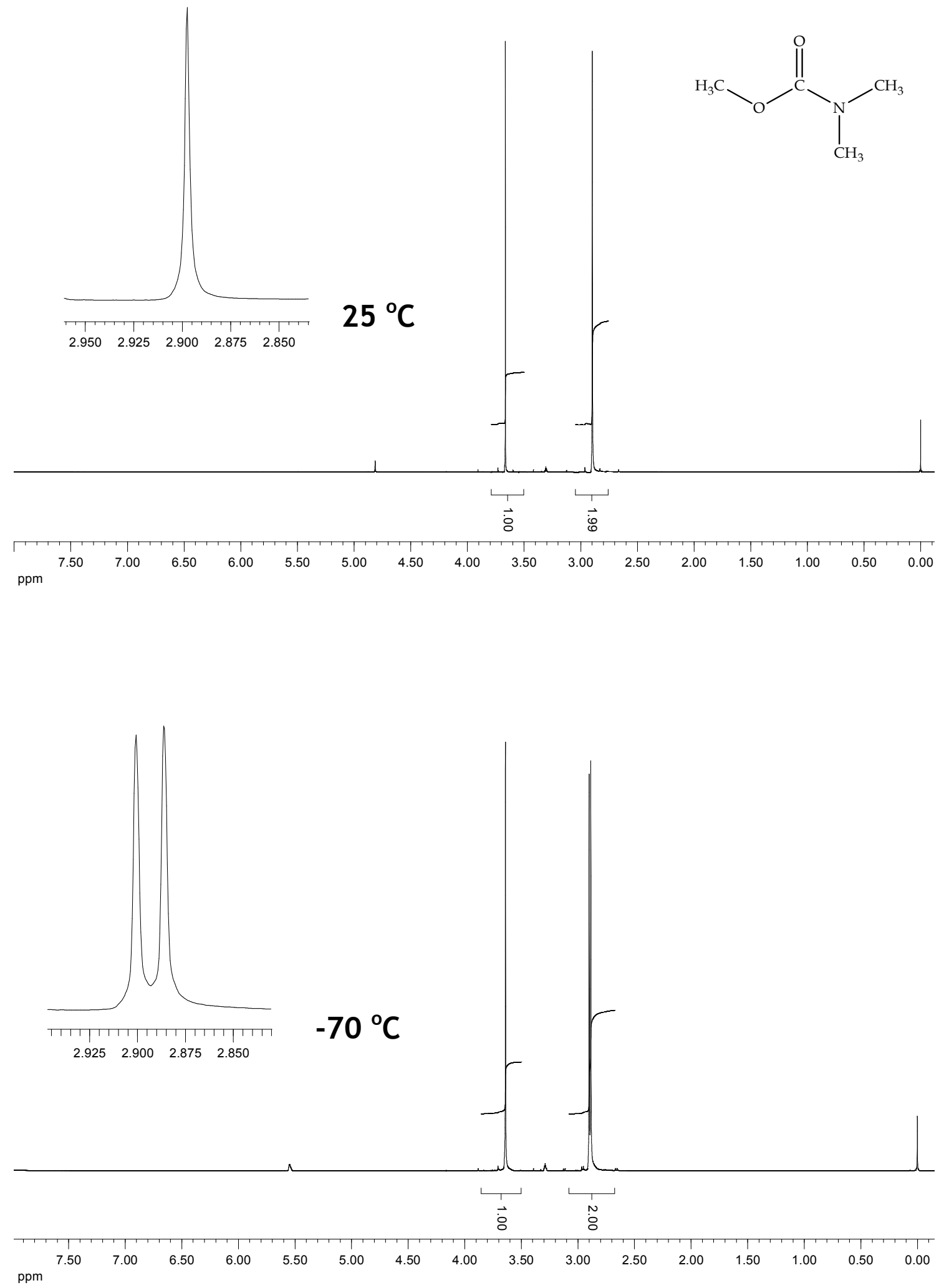

Figure S1. ${ }^{1} \mathrm{H}$ NMR Spectra for methyl $\mathrm{N}, \mathrm{N}$-dimethylcarbamate (1) in $\mathrm{CD}_{3} \mathrm{OD}$ at $300 \mathrm{MHz}$. 

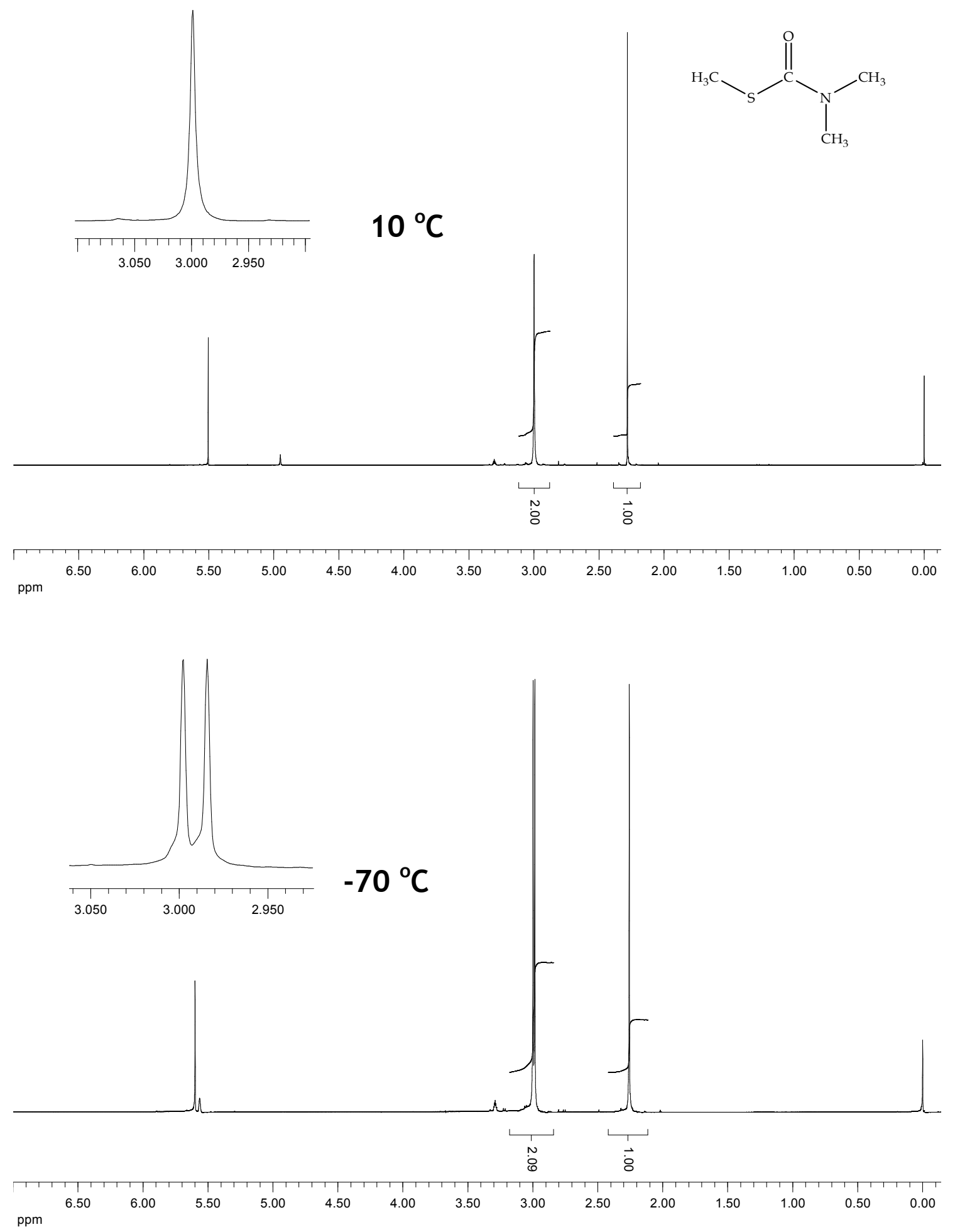

Figure S2. ${ }^{1} \mathrm{H}$ NMR Spectra for S-methyl $\mathrm{N}, \mathrm{N}$-dimethylthiocarbamate (2) in $\mathrm{CD}_{3} \mathrm{OD}$ at $300 \mathrm{MHz}$. 

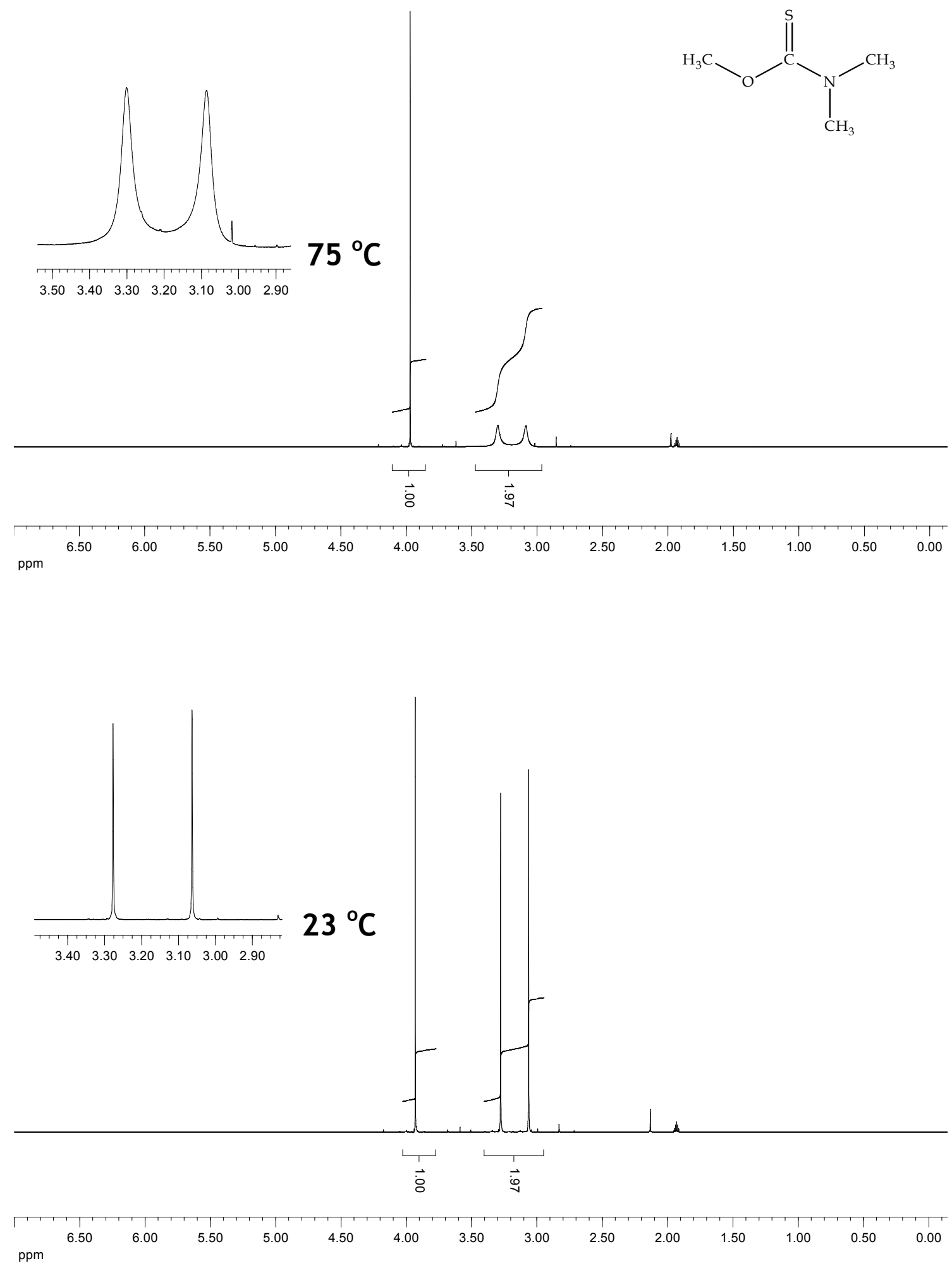

Figure S3. ${ }^{1} \mathrm{H}$ NMR Spectra for O-methyl N,N-dimethylthiocarbamate (3) in $\mathrm{CD}_{3} \mathrm{CN}$ at $300 \mathrm{MHz}$. 

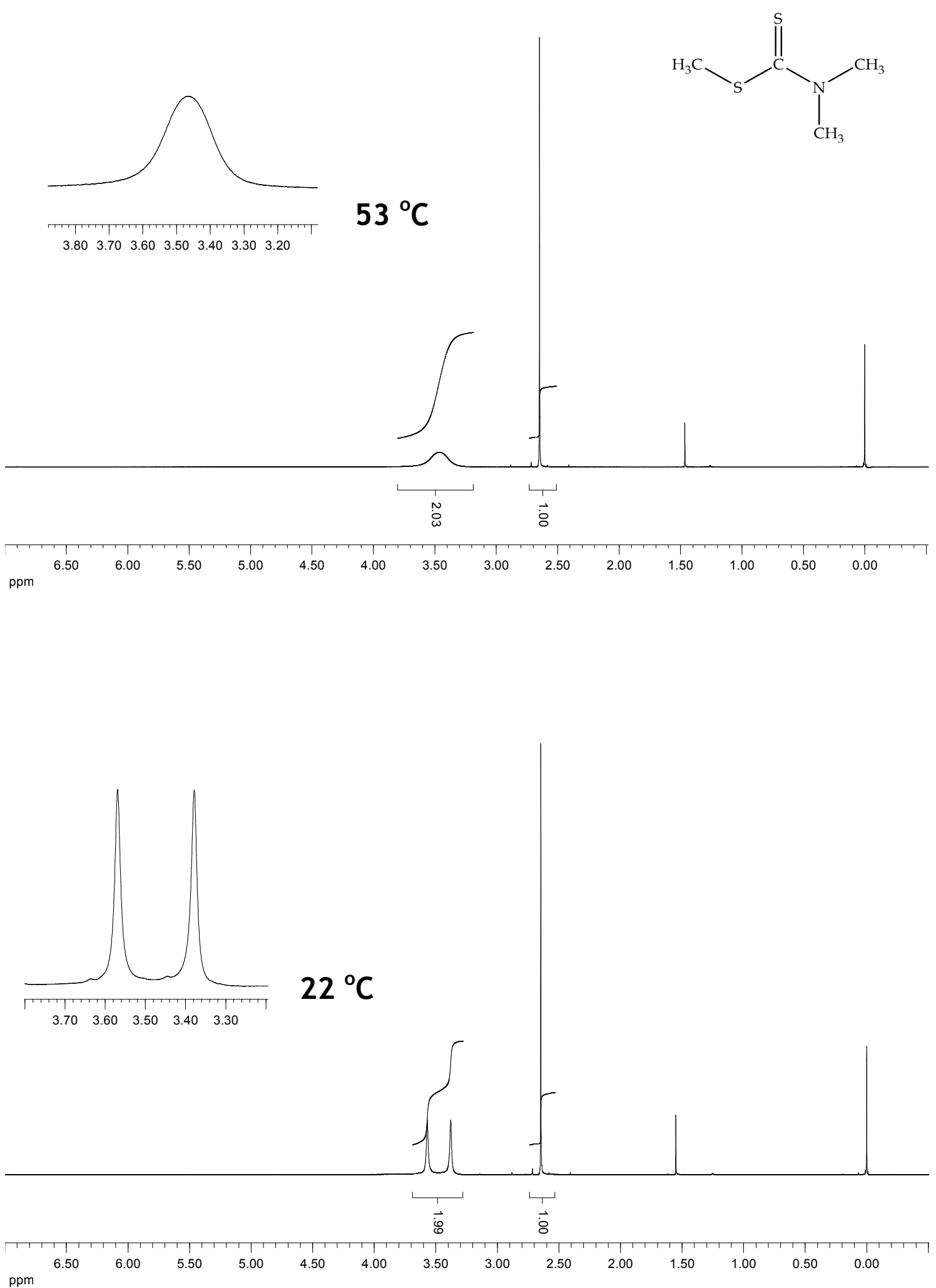

Figure S4. ${ }^{1} \mathrm{H}$ NMR Spectra for methyl N,N-dimethyldithiocarbamate (4) in $\mathrm{CDCl}_{3}$ at $300 \mathrm{MHz}$. 

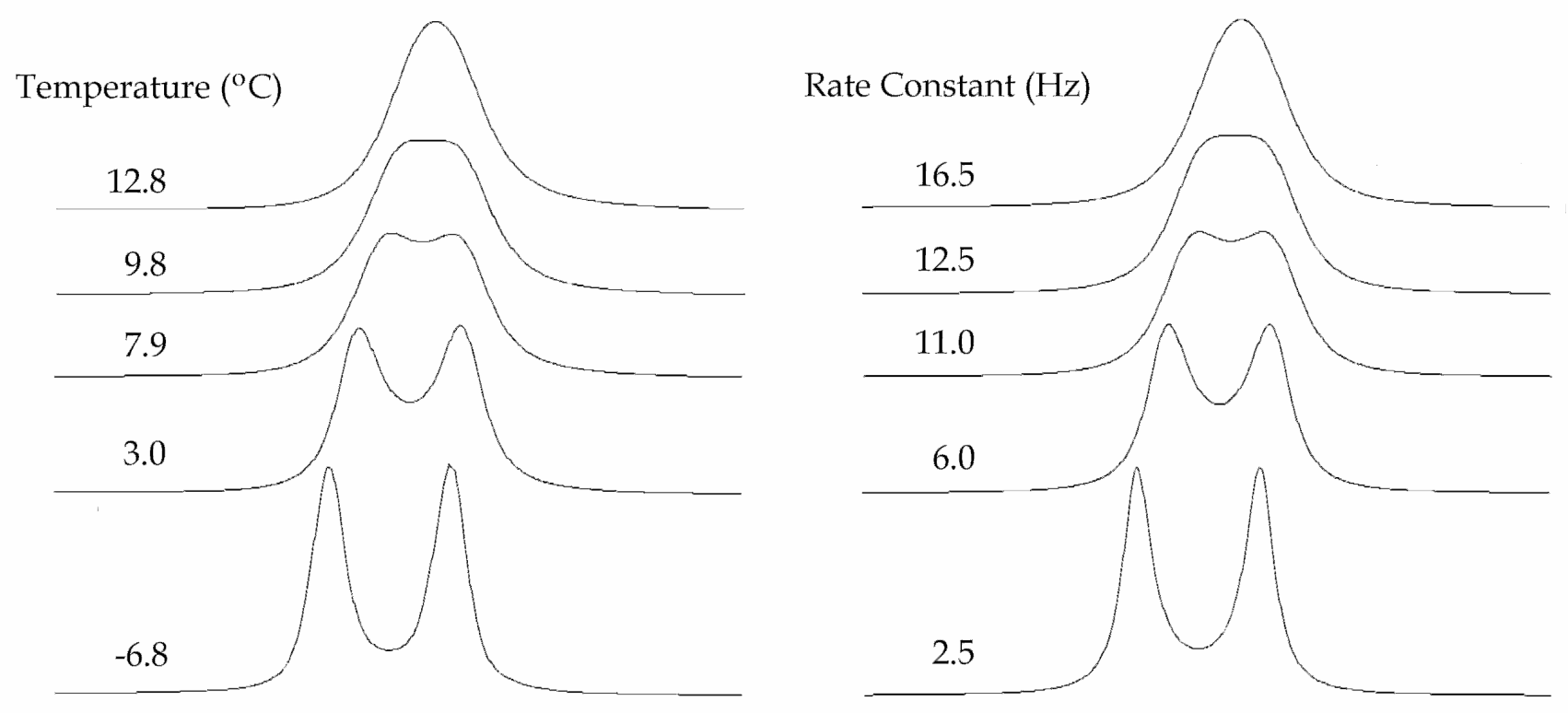

Figure S5. Experimental (left) and simulated (right) ${ }^{1} \mathrm{H}$ NMR spectra for compound 1 in methanol.

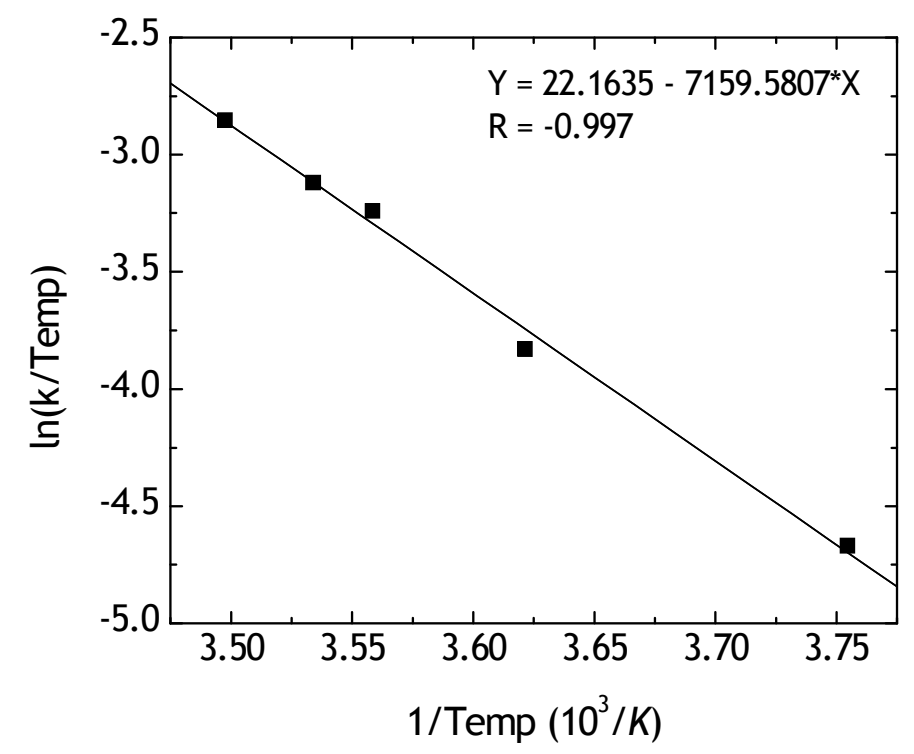

Figure S6. Eyring plot for compound 1 in methanol. 
Table S1. Parameters Used in Molecular Dynamics Simulations *

\begin{tabular}{|c|c|c|c|c|c|}
\hline Atom & $\sigma(\AA)$ & $\varepsilon(\mathrm{kcal} / \mathrm{mol})$ & $q(\mathrm{GS})(e)$ & $q(\mathrm{TS} 1)(e)$ & $q(\mathrm{TS} 2)(e)$ \\
\hline \multicolumn{6}{|c|}{ Methyl N,N-Dimethylcarbamate (1) } \\
\hline $\mathrm{CH}_{3}(-\mathrm{O}-)$ & 3.775 & 0.207 & 0.300 & 0.318 & 0.243 \\
\hline $\mathrm{CH}_{3}(-\mathrm{N}-)$ & 3.800 & 0.170 & 0.125 & 0.267 & 0.265 \\
\hline$C(C=0)$ & 3.750 & 0.105 & 0.200 & 0.360 & 0.380 \\
\hline$O(C=0)$ & 2.960 & 0.210 & -0.400 & -0.353 & -0.353 \\
\hline $0(-0-)$ & 3.070 & 0.170 & -0.280 & -0.255 & -0.156 \\
\hline $\mathrm{N}$ & 3.250 & 0.170 & -0.070 & -0.604 & -0.644 \\
\hline \multicolumn{6}{|c|}{ S-Methyl N,N-Dimethylthiocarbamate (2) } \\
\hline $\mathrm{CH}_{3}(-\mathrm{S}-)$ & 3.800 & 0.177 & 0.230 & 0.200 & 0.200 \\
\hline $\mathrm{CH}_{3}(-\mathrm{N}-)$ & 3.800 & 0.170 & 0.140 & 0.235 & 0.280 \\
\hline$C(C=0)$ & 3.750 & 0.105 & 0.225 & 0.398 & 0.380 \\
\hline$O(C=0)$ & 2.960 & 0.210 & -0.400 & -0.353 & -0.340 \\
\hline$S(-S-)$ & 3.550 & 0.250 & -0.270 & -0.211 & -0.166 \\
\hline $\mathrm{N}$ & 3.250 & 0.170 & -0.065 & -0.504 & -0.634 \\
\hline \multicolumn{6}{|c|}{ O-Methyl N,N-Dimethylthiocarbamate (3) } \\
\hline $\mathrm{CH}_{3}(-\mathrm{O}-)$ & 3.070 & 0.170 & -0.122 & 0.243 & 0.270 \\
\hline $\mathrm{CH}_{3}(-\mathrm{N}-)$ & 3.800 & 0.170 & 0.090 & 0.192 & 0.225 \\
\hline$C(C=S)$ & 3.750 & 0.105 & 0.060 & 0.303 & 0.342 \\
\hline$S(C=S)$ & 3.550 & 0.250 & -0.263 & -0.210 & -0.190 \\
\hline $0(-0-)$ & 3.070 & 0.170 & -0.122 & -0.160 & -0.167 \\
\hline $\mathrm{N}$ & 3.250 & 0.170 & -0.055 & -0.560 & -0.705 \\
\hline \multicolumn{6}{|c|}{ S-Methyl N,N-Dimethyldithiocarbamate (4) } \\
\hline $\mathrm{CH}_{3}(-\mathrm{S}-)$ & 3.800 & 0.177 & 0.160 & 0.148 & 0.183 \\
\hline $\mathrm{CH}_{3}(-\mathrm{N}-)$ & 3.800 & 0.170 & 0.130 & 0.180 & 0.265 \\
\hline$C(C=S)$ & 3.750 & 0.105 & 0.200 & 0.290 & 0.240 \\
\hline$S(C=S)$ & 3.550 & 0.250 & -0.270 & -0.213 & -0.153 \\
\hline$S(-S-)$ & 3.550 & 0.250 & -0.280 & -0.145 & -0.156 \\
\hline $\mathrm{N}$ & 3.250 & 0.170 & -0.070 & -0.440 & -0.644 \\
\hline
\end{tabular}

* Lennard-Jones from Refs. 11, 44-47. 